$\underline{\text { Supporting Information }}$

\title{
Computational Methods to Predict the Regioselectivity of Electrophilic Aromatic Substitution Reactions of Heteroaromatic Systems.
}

Monika Kruszyk, ${ }^{\dagger}, \S$ Mikkel Jessing, ${ }^{\dagger}$ Jesper Langgaard Kristensen, ${ }^{\S}$ and Morten Jørgensen $^{\dagger} * *$

† Discovery Chemistry and DMPK, Neuroscience Drug Discovery, H. Lundbeck A/S, Ottiliavej 9, 2500 Valby, Denmark

${ }^{\S}$ Department of Drug Design and Pharmacology, Faculty of Health and Medical Science, University of Copenhagen, Universitetsparken 2, 2100 Copenhagen $\emptyset$, Denmark

*Corresponding Author, E-mail: mojj@lundbeck.com

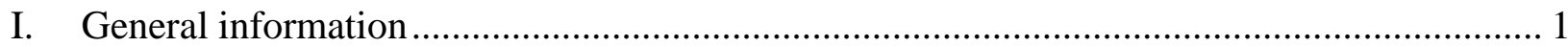

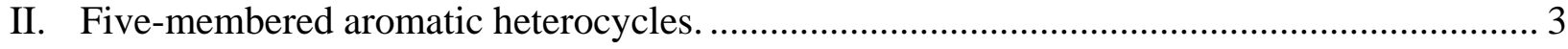

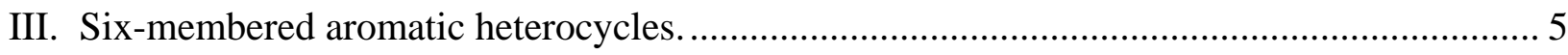

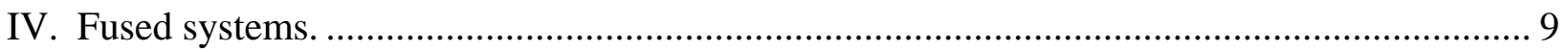

V. The HOMO graphical models of compounds 17 and 119-136 .................................... 10

VI. Cartesian coordinates and total energy values for optimized geometries of compounds $\mathbf{1 7}$, 20, 48, 49, 119-136. 15

I. General information

The ${ }^{13} \mathrm{C}$ and/or ${ }^{1} \mathrm{H}$ chemical shift predictions have been calculated using ChemDraw from PerkinElmer®, version currently available at the time the predictions were performed (14.0 and higher). The ${ }^{1} \mathrm{H}$ and ${ }^{13} \mathrm{C}$ values are represented in green and red, respectively. For the ${ }^{1} \mathrm{H}$ spectrum the variation of $0.1 \mathrm{ppm}$ and for ${ }^{13} \mathrm{C}$ spectrum the variation of $5 \mathrm{ppm}$ relative to the lowest predicted value is accepted. The circles point to the most reactive site according to the 
predictions, while the arrow indicates the site of the actual reaction. For simplicity, the values for inactivated and/or deactivated benzenes are omitted.

The quantum mechanical calculations were performed by the Jaguar software using DFT with B3LYP density functional model in the gas phase (Jaguar, Version 8.9, Schrödinger, LLC, New York, NY, 2015). ${ }^{1}$ The geometries were optimized using the $6-31 G^{* *}$ basis set. The HOMO orbitals were represented alone and mapped onto electron density isosurface and are considered as the determinig factor for the regioselectivity. On the graphical models red suggests the site of the potential reaction. 
II. Five-membered aromatic heterocycles.

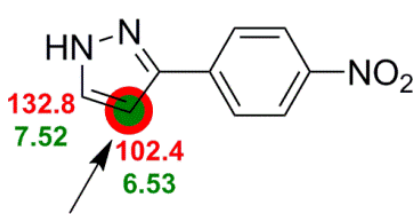

$50,90 \%(N B S)^{2}$

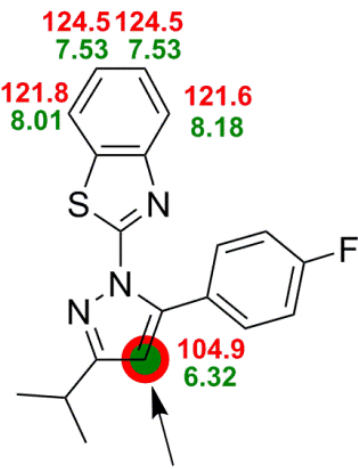

53, $91 \%(N B S)^{5}$

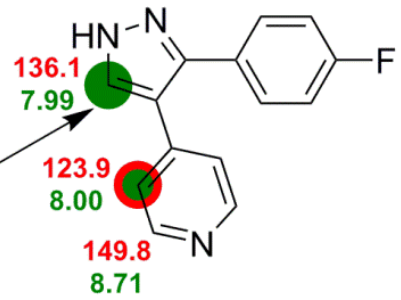

56, $72 \%(N B S)^{6}$

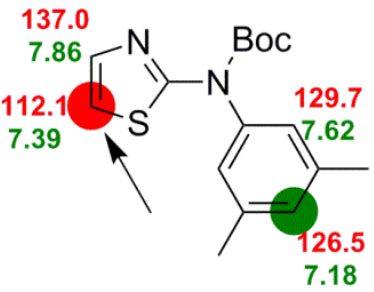

59, $90 \%(N B S)^{9}$

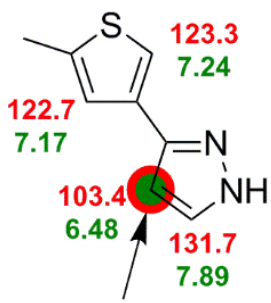

51, $55 \%(N B S)^{3}$

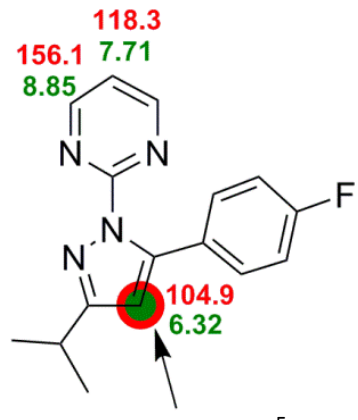

54, $83 \%(N B S)^{5}$

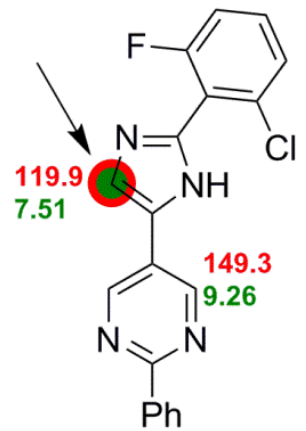

$\mathbf{5 7}$, No yields reported (NBS) ${ }^{7}$

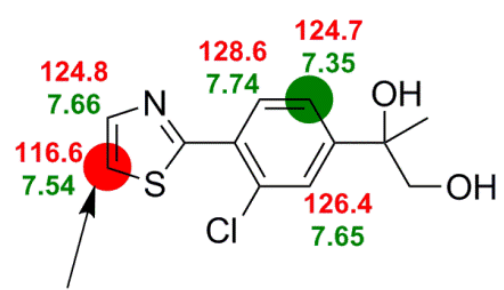

$60,100 \%(N B S)^{10}$

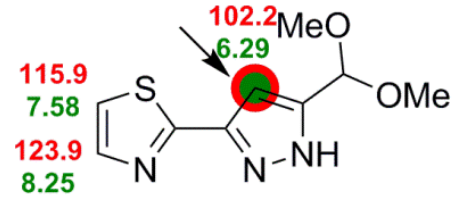

52, $93 \%\left(\mathrm{Br}_{2}, \mathrm{AcOH}\right)^{4}$

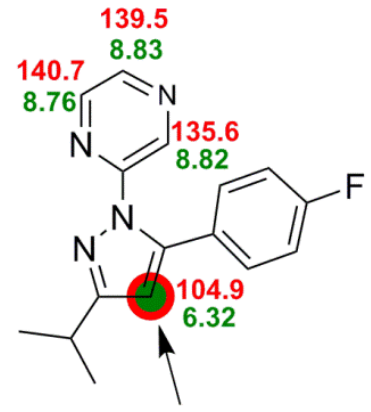

$55,88 \%(N B S)^{5}$

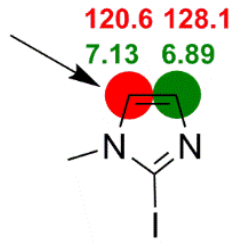

58, $87 \%(N B S)^{8}$

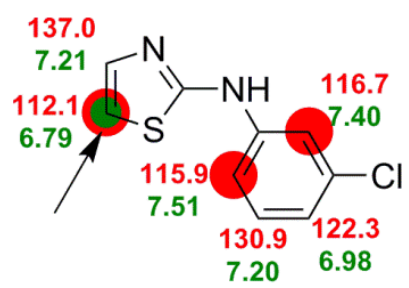

61, 65\% $\left(\mathrm{Br}_{2}, \mathrm{AcOH}\right)^{11}$ 


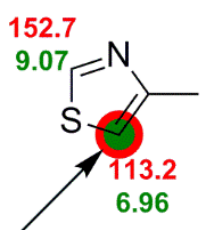

62, 37\% $\left(\mathrm{Br}_{2}, \mathrm{AcOH}\right)^{12}$

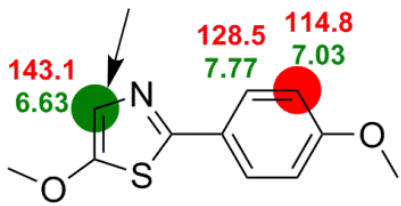

$65,80 \%(N B S)^{15}$

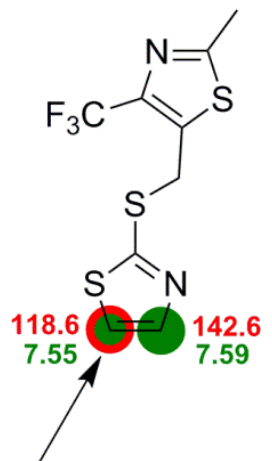

67a, 96\% (NBS)

67b, 63\% (NIS)

67c, $73 \%\left(\mathrm{HNO}_{3}\right)^{17}$

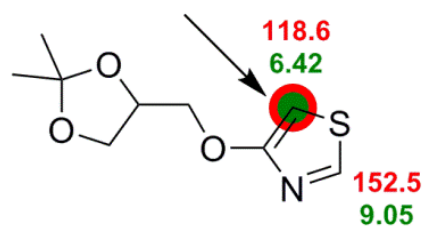

63, No yields (NBS) ${ }^{13}$

149.2

8.74

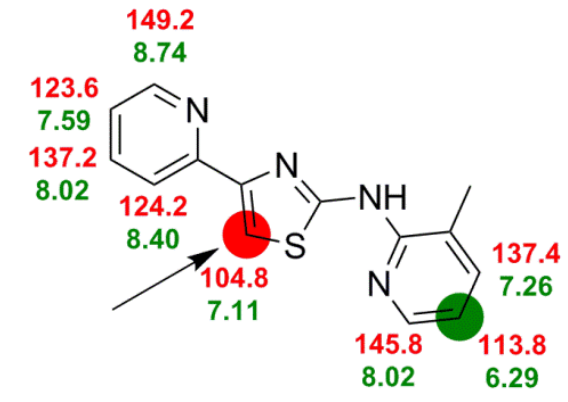

66a, 86\% (NBS)

66b, 93\% (NCS) ${ }^{16}$

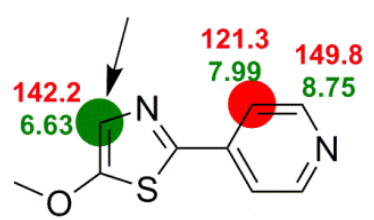

$64,96 \%(N B S)^{14}$ 
III. Six-membered aromatic heterocycles.

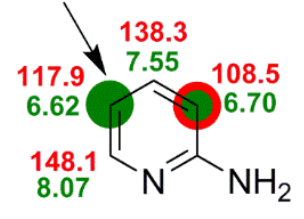

$68,98 \%(N B S)^{18}$

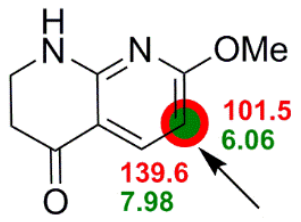

$71,85 \%$ (NBS) $^{20}$

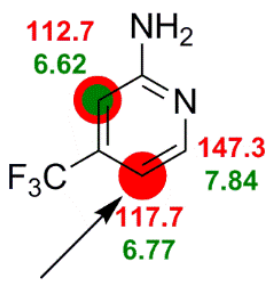

$69,80 \%(N B S)^{19}$

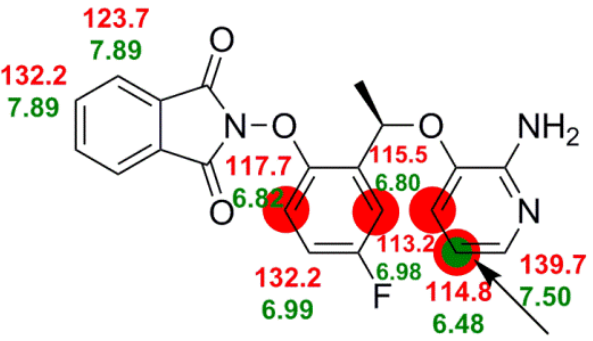

72, No yields reported (NBS) ${ }^{21}$

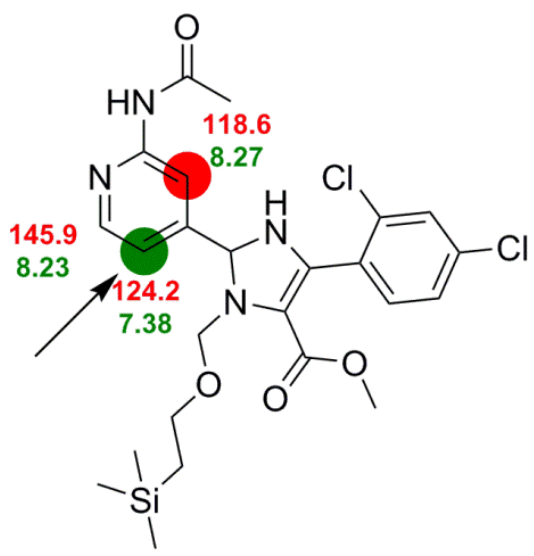

$75,87 \%$ (NBS) $^{24}$

77, $90 \%$ (NBS) $^{26}$
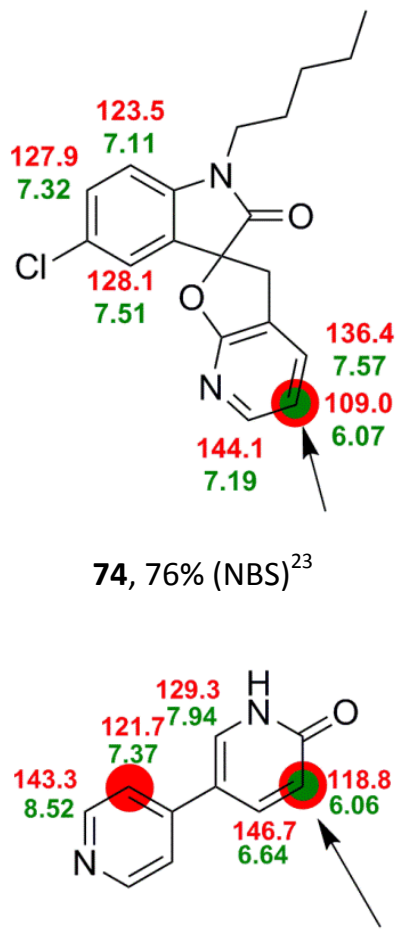

74, $76 \%$ (NBS) $^{23}$

\footnotetext{
.
}




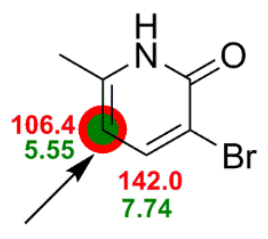

$\mathbf{8 0}, 91 \%(\mathrm{NIS})^{30}$

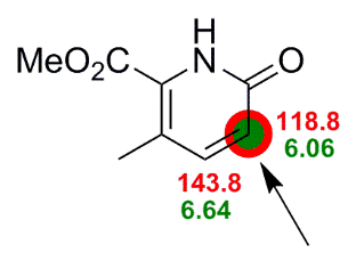

83, $70 \%(N B S)^{33}$

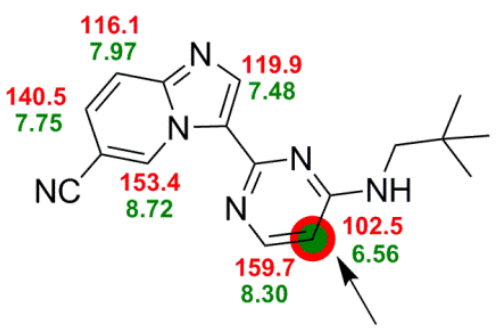

$86,98 \%$ (NBS) $^{36}$

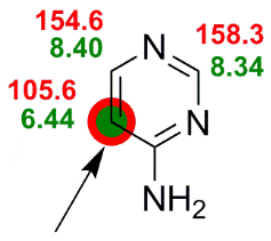

$89,59 \%$ (NBS) $^{39}$

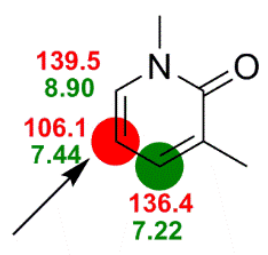

$\mathbf{8 1}, 96 \%$ (NBS) $^{31}$

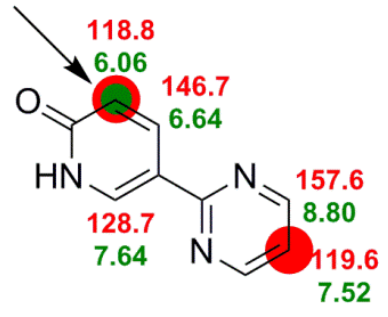

$84,51 \%(N B S)^{34}$

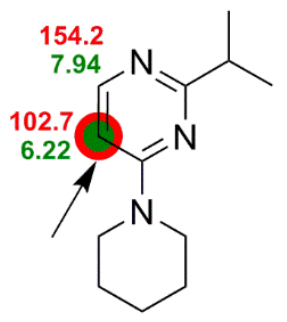

$87,82 \%$ (NBS) $^{37}$

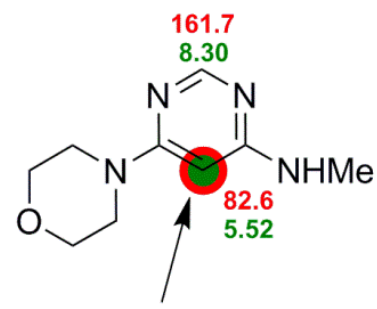

90, $78 \%\left(\mathrm{Br}_{2}\right)^{40}$

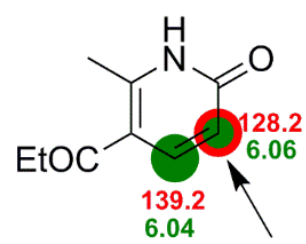

$82,82 \%$ (NBS) $^{32}$

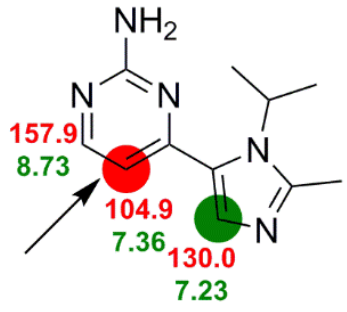

$85,74 \%(\mathrm{NIS})^{35}$

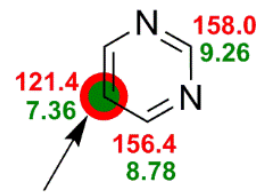

( $\mathrm{HCl}$ salt)88, 88\% $\left(\mathrm{Br}_{2}\right)^{38}$

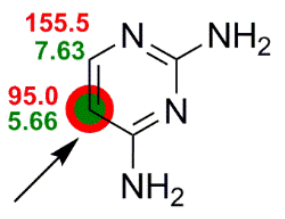

91, 74\% (NBS) ${ }^{19}, 87 \%\left(\mathrm{Br}_{2}\right)^{41}$ 


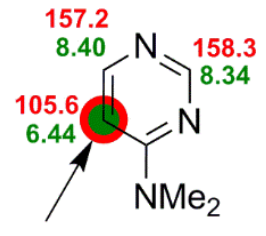

92, $91 \%$ (NBS) $^{42}$

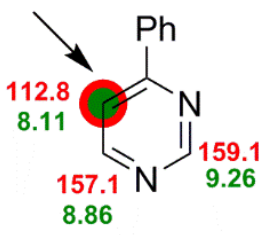

(HCl salt)95, 41\% (98\% BRSM, NBS) ${ }^{44}$

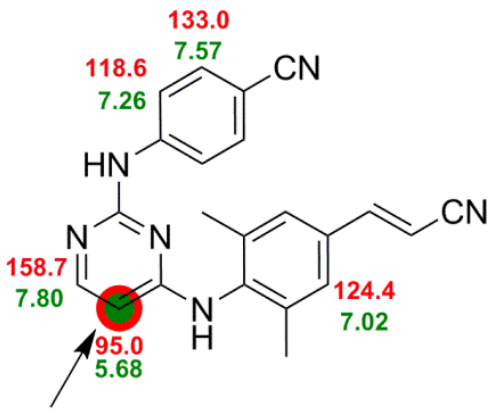

$98,86 \%\left(\right.$ NBS $^{46}$

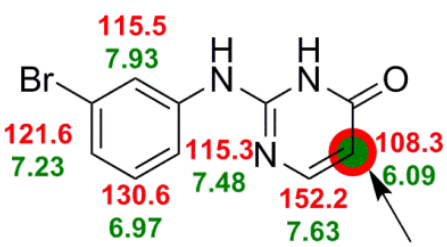

101a, $82 \%$ (NCS)

101b, 73\% (NBS)

101c, $97 \%(\mathrm{NIS})^{50}$

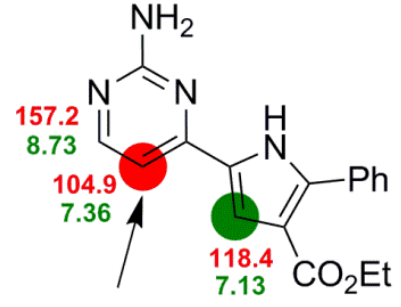

93a, 58\% (NCS)

93b, 65\% (NBS) ${ }^{43}$

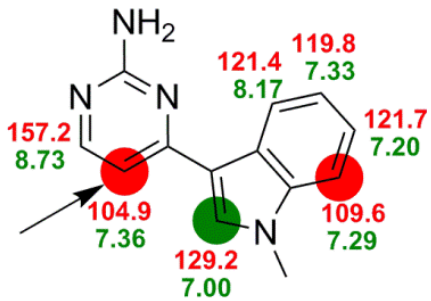

$96,94 \%$ (NBS) $^{45}$

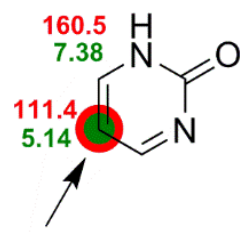

99a, 50\% $\left(\mathrm{Br}_{2}\right)^{47}$ ( $\mathrm{HCl}$ salt) 99b, 84\% $\left(\mathrm{Br}_{2}\right)^{48}$

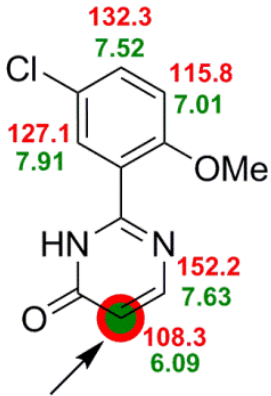

102, $86 \%\left(\mathrm{Br}_{2}\right)^{51}$

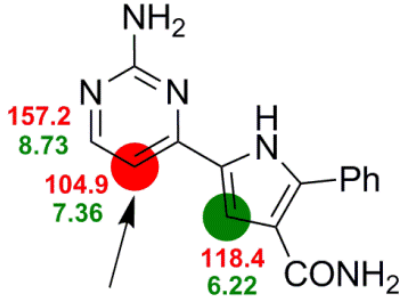

94a, 72\% (NCS)

94b, 75\% (NBS) ${ }^{43}$

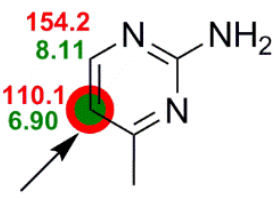

97, $99 \%$ (NBS) $^{19}$

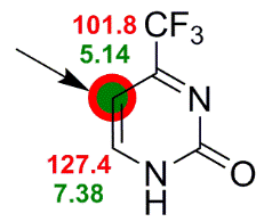

$100,80 \%\left(\mathrm{Br}_{2}, \mathrm{KOAC}\right)^{49}$

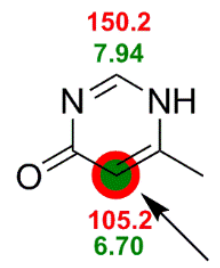

103a, 74\% (NCS)

103b, $94 \%(N B S)^{52}$

103c, $100 \%(\mathrm{ICl}, \mathrm{AcOH})^{53}$ 


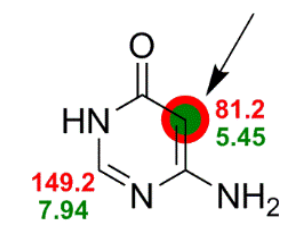

104, $47 \%\left(\mathrm{Br}_{2}\right)^{54}$

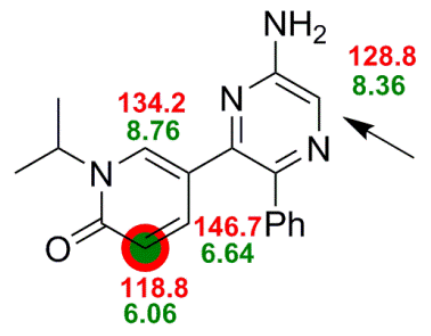

$107,79 \%(N B S)^{57}$

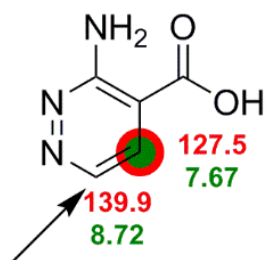

110 , no yields (NIS) ${ }^{60}$

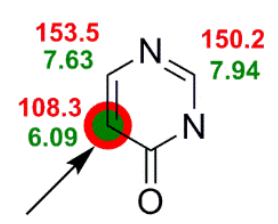

105, $92 \%\left(\mathrm{Br}_{2}\right)^{55}$

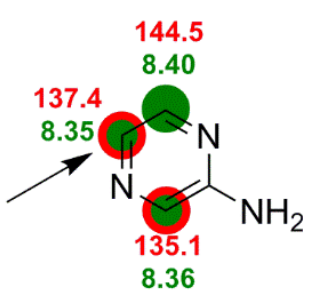

$108,82 \%(N B S)^{58}$

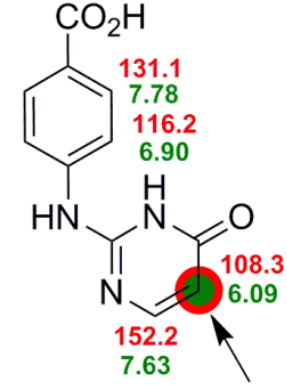

106, $95 \%\left(\mathrm{Br}_{2}, \mathrm{AcOH}\right)^{56}$

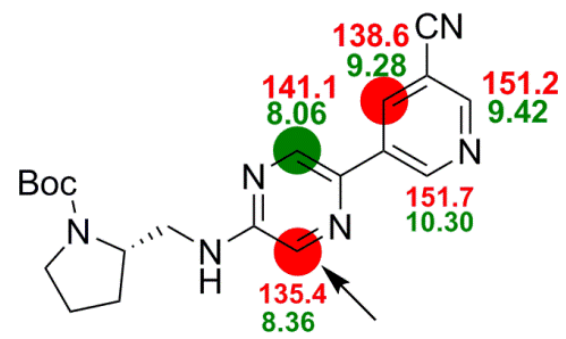

$109,66 \%$ (NBS) $^{59}$ 
IV. Fused systems.

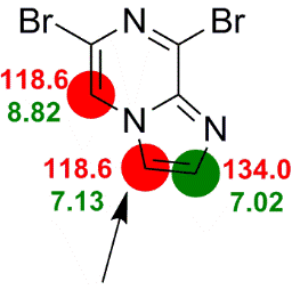

111a, No yields reported (NBS) ${ }^{61}$ 111b, $75 \%(N I S)^{62}$

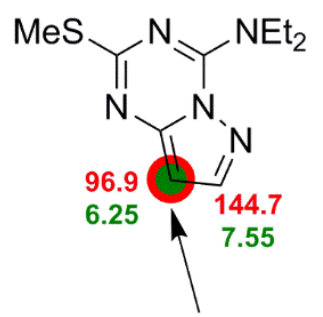

$114,75 \%(N B S)^{64}$

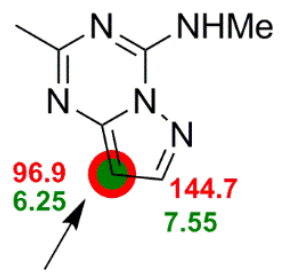

117, $73 \%\left(\mathrm{Br}_{2}, \mathrm{AcOH}\right)^{67}$

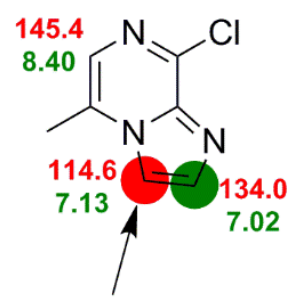

$112,69 \%(N B S)^{63}$

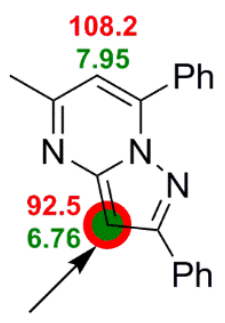

115a, 87\% (NBS)

115b, 70\% (NIS) ${ }^{65}$

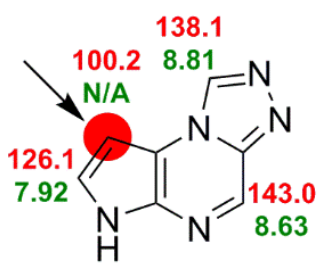

$118,57 \%(N I S)^{68}$

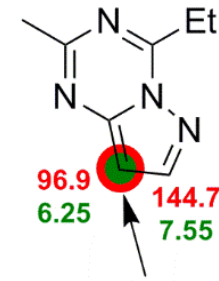

113, $41 \%(N B S)^{64}$

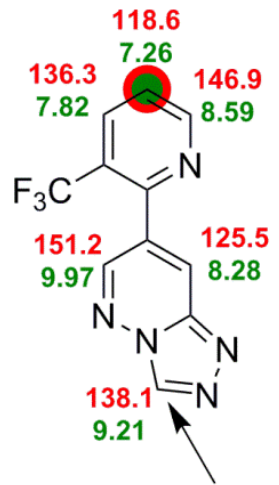

$116,100 \%\left(\mathrm{Br}_{2}, \mathrm{AcOH}\right)^{66}$ 
V. The HOMO graphical models of compounds 17 and 119-136.

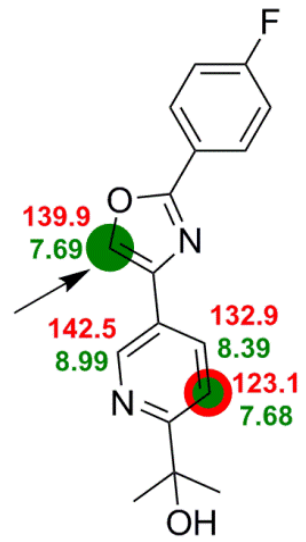

$119,66 \%(N B S)^{69}$

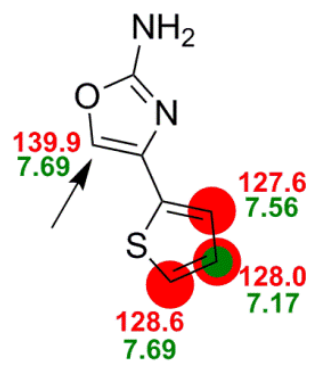

120, No yields reported (NBS) $)^{70}$

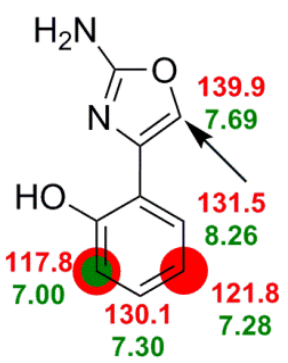

121, No yields reported (NBS) $)^{70}$
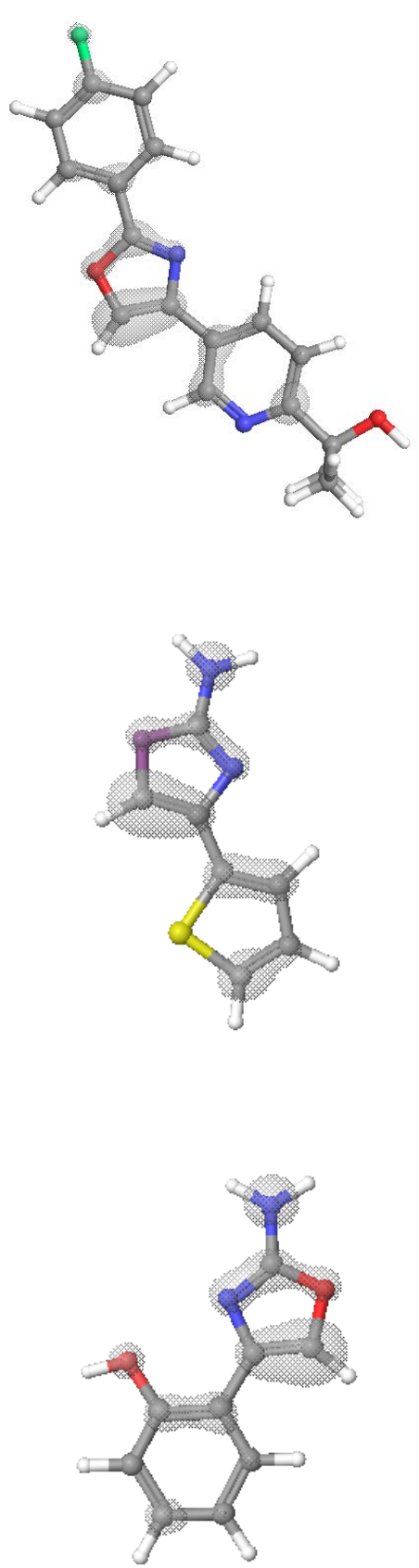
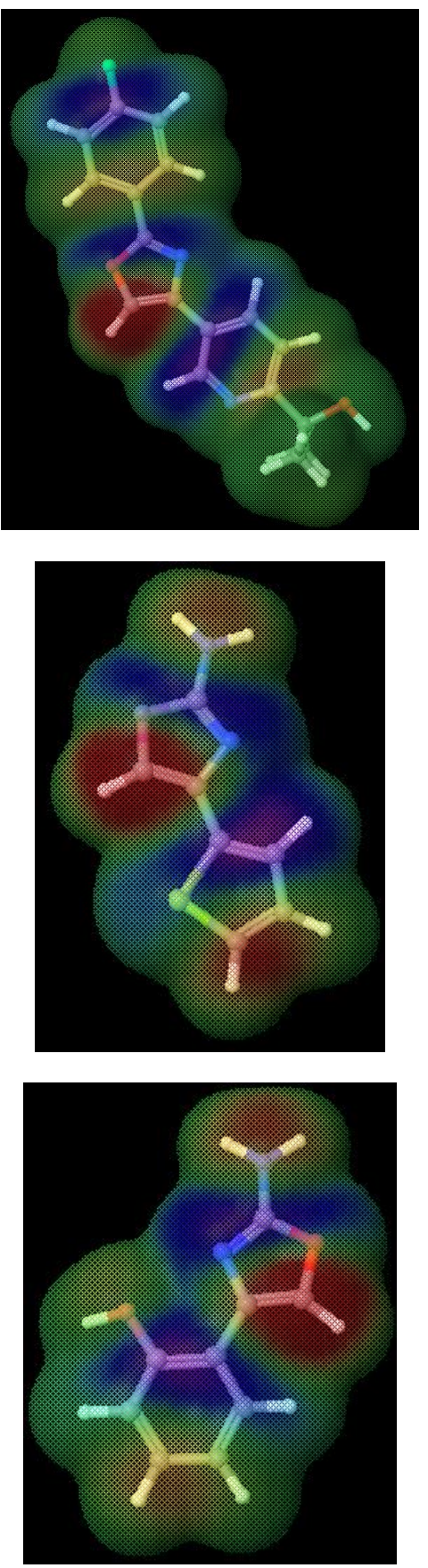


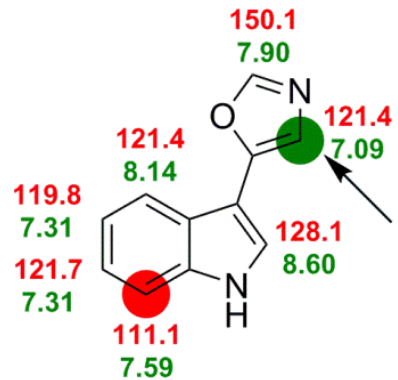

$122,84 \%(N B S)^{71}$

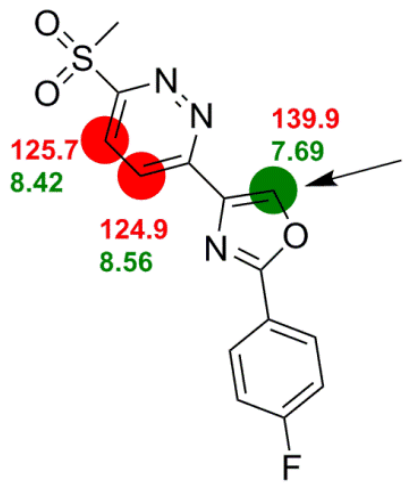

123, No yields reported (NBS) ${ }^{72}$

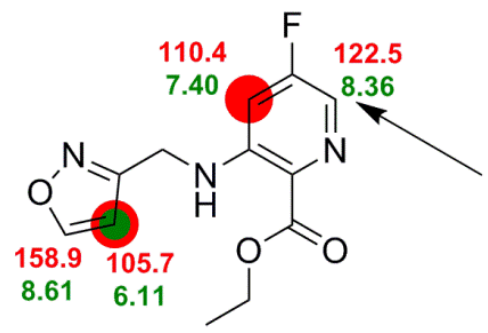

124, $34 \%(N B S)^{73}$
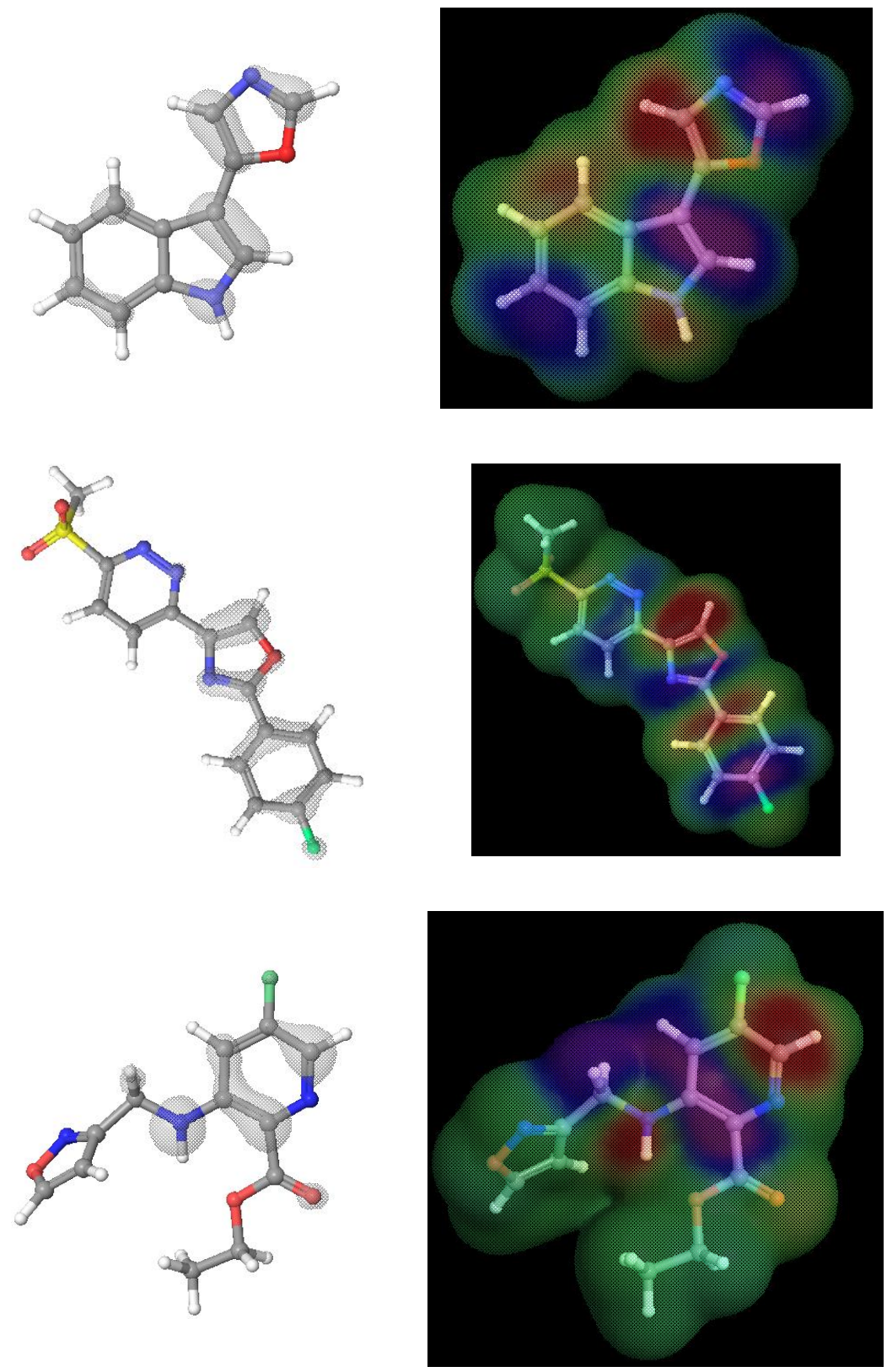


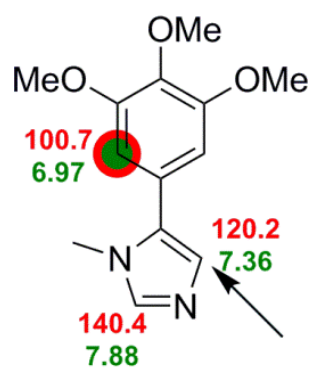

$17,80 \%$ (NBS) $^{74}$

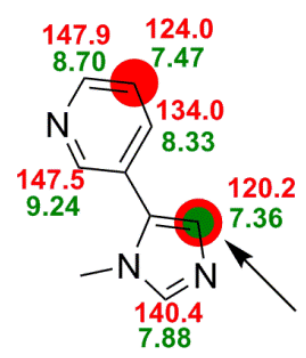

$125,71 \%(N B S)^{74}$

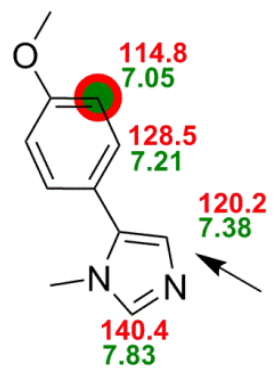

126, $55 \%\left(\right.$ NBS $^{74}$
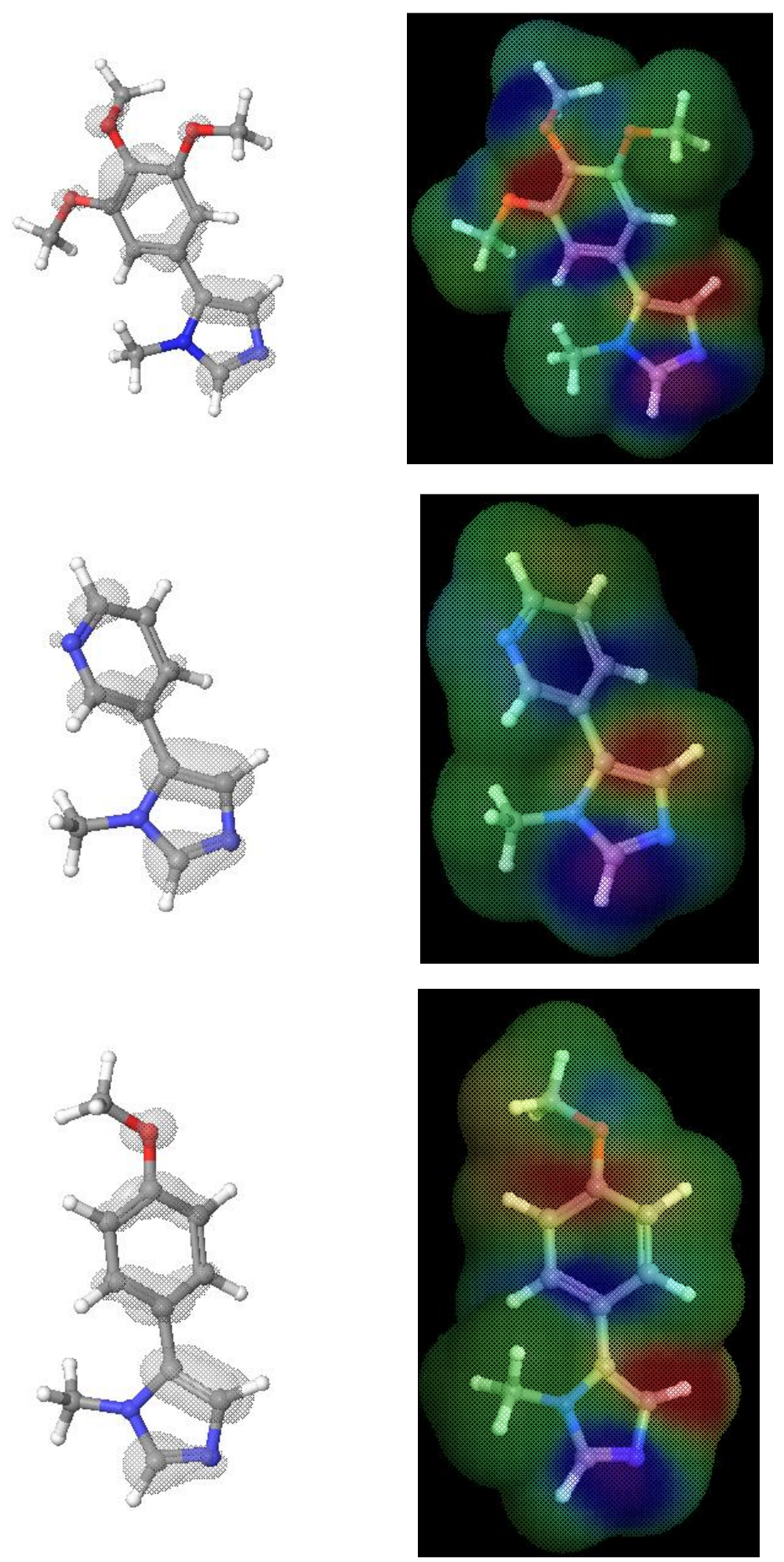


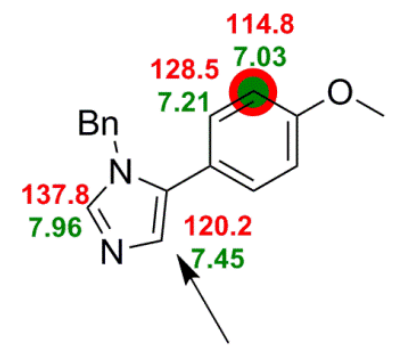

127a, $80 \%$ (NBS) $^{75}$

127b, $84 \%$ (NIS, TFA) ${ }^{76}$

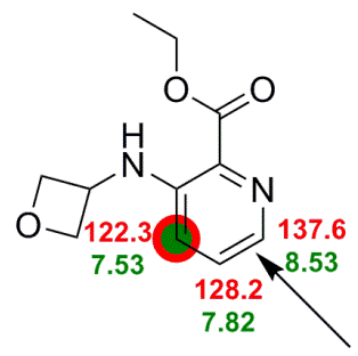

$128,76 \%$ (NBS) $^{73}$

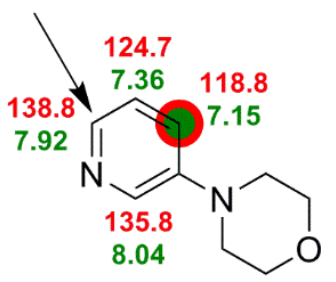

$129,74 \%(N B S)^{77}$

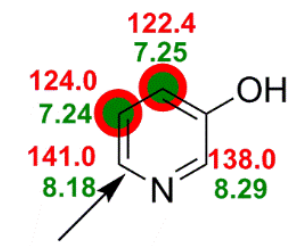

130, 75\% (Nal, $\mathrm{NaOCl})^{78}$
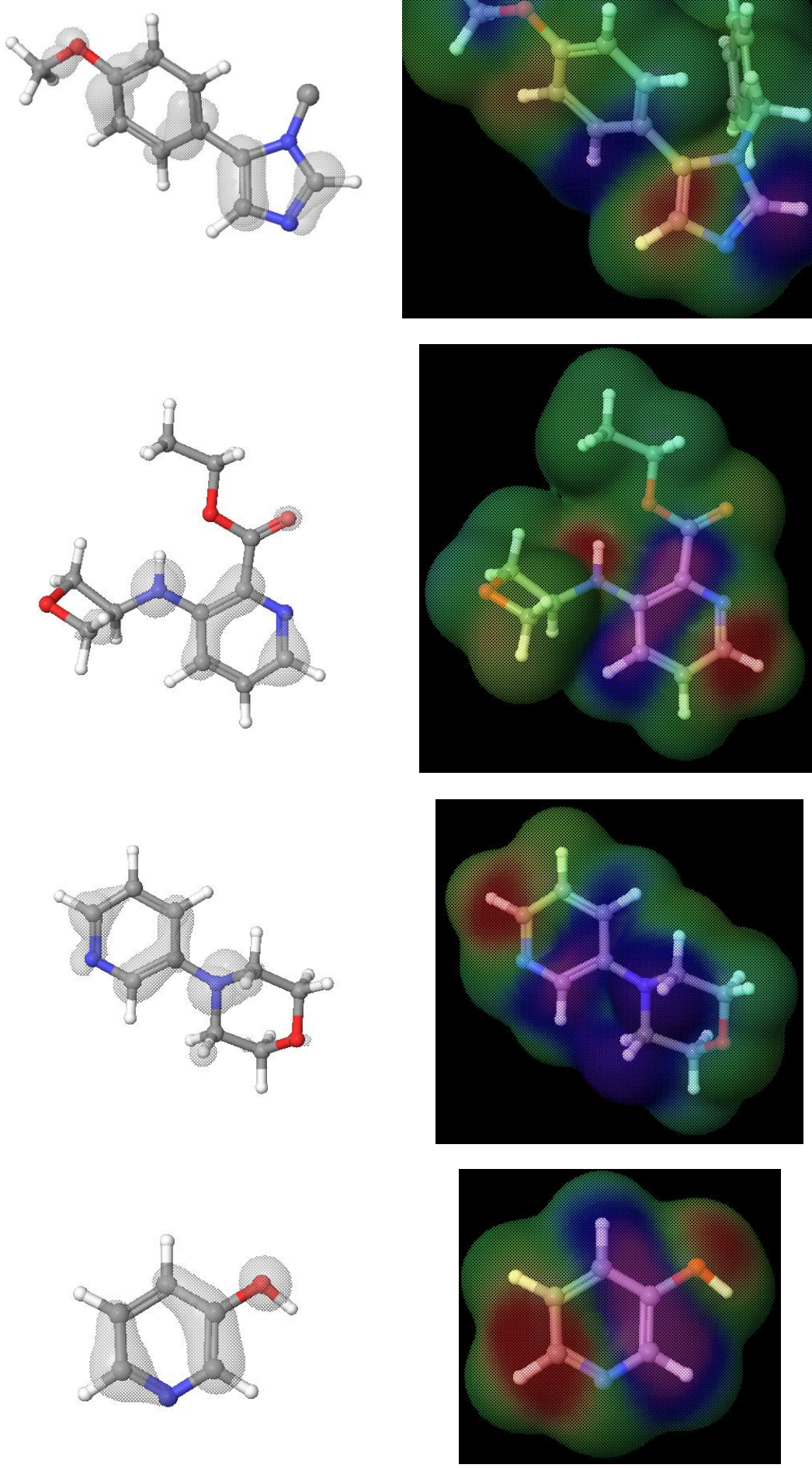


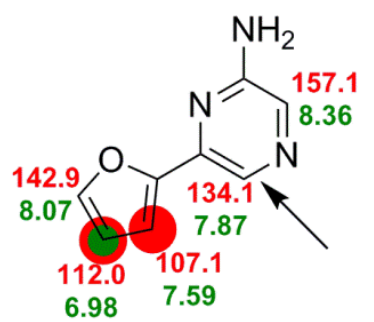

131, $44 \%(N B S)^{79}$

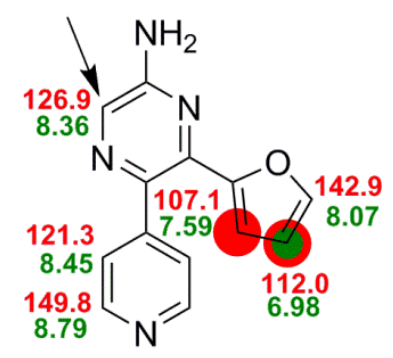

$132,75 \%$ (NBS) $^{79}$

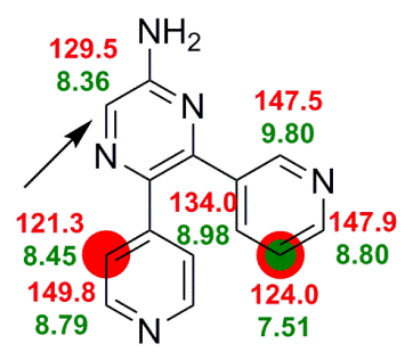

133, 30\%(NBS) $)^{79}$

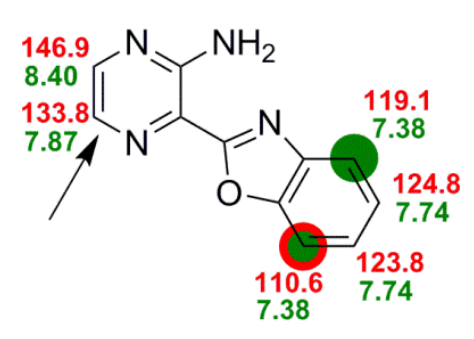

$134,62 \%$ (NBS) $^{80}$
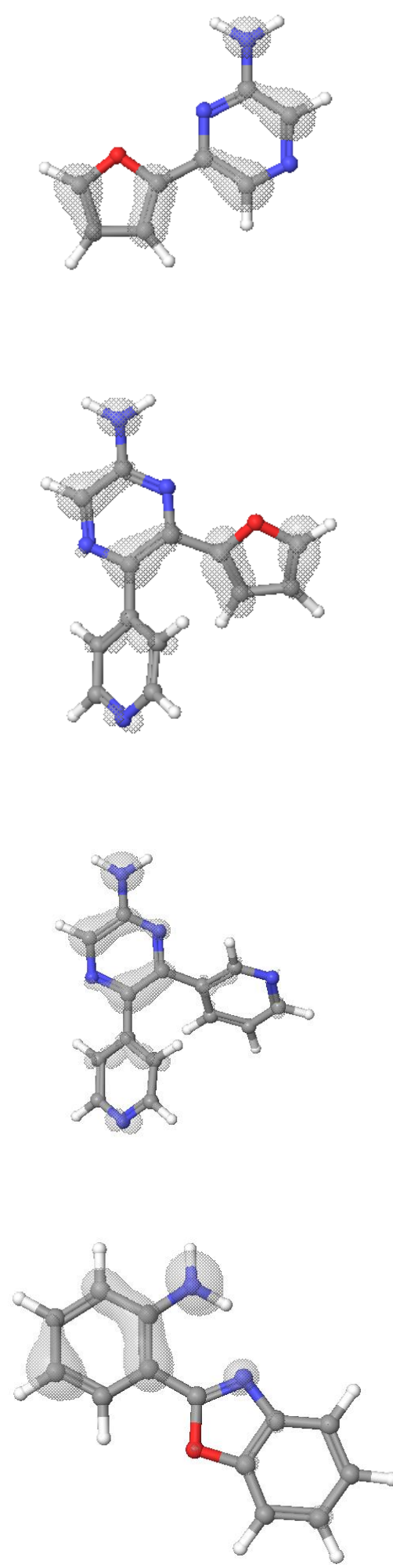
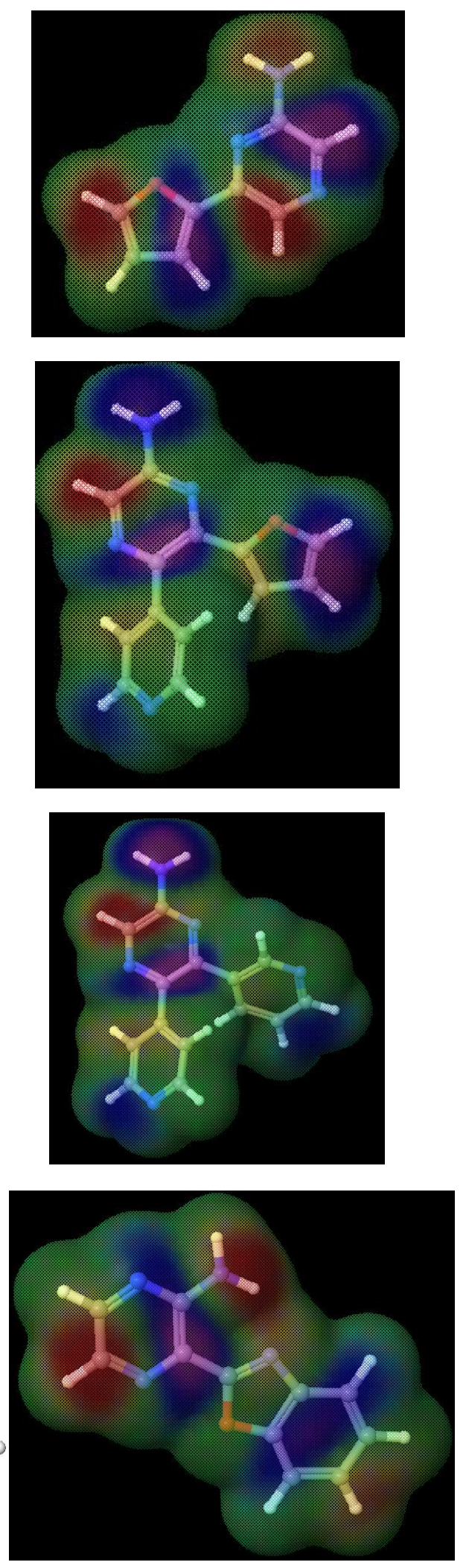


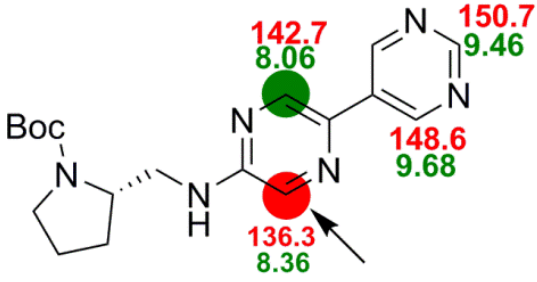

$135^{1}, 77 \%(\mathrm{NBS})^{59}$

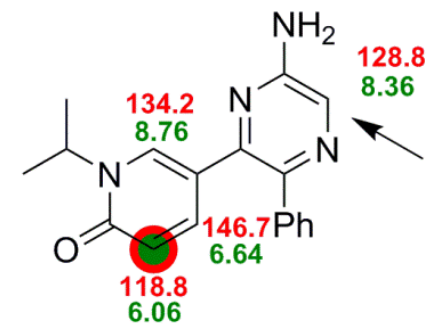

$136,79 \%(N B S)^{57}$
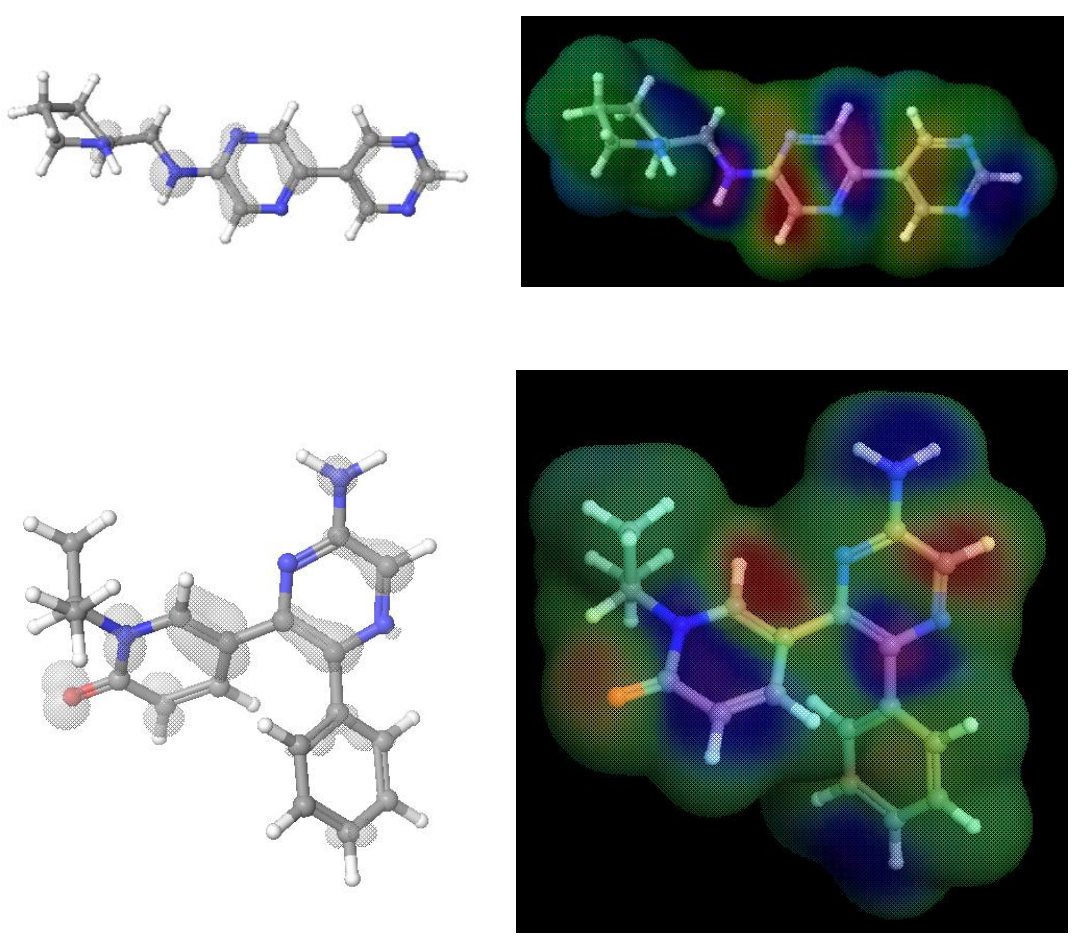

VI. Cartesian coordinates and total energy values for optimized geometries of compounds $\mathbf{1 7}$, 20, 48, 49, 119-136.

17:

$\mathbf{X}$

$\mathrm{N} \quad-1.87250$

$-1.04640$

$-0.00180$

$-0.14720$

$-1.29520$

$-1.26340$

0.56140

$-2.75720$

$-3.65640$

$-3.19670$

$-1.83010$

$-0.92810$

$-1.38680$

$-3.22770$

$-4.95870$

$-3.88510$

\section{angstroms}

\section{$y$}

$-1.66900$

$-2.67590$

$-2.23250$

$-0.86910$

$-0.47670$

$-3.71280$

$-0.22650$

3.49690

2.47030

1.16370

0.86980

1.89570

3.20200

4.75620

2.85720

0.38750

\section{$\mathbf{z}$}

1.97760

1.55700

0.89210

0.88440

1.54700

1.77810

0.38060

2.19520

1.86170

1.65890

1.78960

2.11230

2.30320

2.46410

1.74180

1.35240

\footnotetext{
${ }^{1}$ For simplicity, the Boc protecting group is omitted in DFT calculations, due to its minor influence on the neigboring aromatic heterocycles.
} 


$\begin{array}{lrrr}\mathrm{H} & 0.11930 & 1.65170 & 2.23190 \\ \mathrm{O} & -0.58810 & 4.26350 & 2.61140 \\ \mathrm{C} & -3.13520 & 5.67200 & 1.37090 \\ \mathrm{C} & 0.80630 & 4.03550 & 2.74950 \\ \mathrm{C} & -5.92180 & 1.87460 & 1.40150 \\ \mathrm{H} & -6.88160 & 2.39280 & 1.37480 \\ \mathrm{H} & -5.72390 & 1.43250 & 0.41610 \\ \mathrm{H} & -5.96710 & 1.07320 & 2.15040 \\ \mathrm{H} & -3.55170 & 6.61700 & 1.72620 \\ \mathrm{H} & -2.09290 & 5.82570 & 1.07000 \\ \mathrm{H} & -3.71950 & 5.32040 & 0.51180 \\ \mathrm{H} & 1.24550 & 5.00560 & 2.98640 \\ \mathrm{H} & 1.02270 & 3.33280 & 3.56380 \\ \mathrm{H} & 1.24970 & 3.65480 & 1.82060 \\ \mathrm{C} & -3.06140 & -1.85210 & 2.79380 \\ \mathrm{H} & -3.13610 & -1.05120 & 3.53110 \\ \mathrm{H} & -3.97440 & -1.85880 & 2.18920 \\ \mathrm{H} & -2.98460 & -2.80770 & 3.31690 \\ \text { Total energy......... } & -840.16323821097 & \text { hartrees } \\ \text { Number of } & \text { imaginary } & \text { frequencies: } 0 & \end{array}$

20:

$\mathrm{X}$

$\begin{array}{ll}\mathrm{C} & -8.96280 \\ \mathrm{~N} & -8.25450 \\ \mathrm{C} & -6.90280 \\ \mathrm{C} & -6.54950 \\ \mathrm{~S} & -7.97630 \\ \mathrm{H} & -6.21260 \\ \mathrm{O} & -5.34800 \\ \mathrm{C} & -13.24440 \\ \mathrm{C} & -12.56270 \\ \mathrm{C} & -11.16760 \\ \mathrm{C} & -10.42800 \\ \mathrm{C} & -11.13270 \\ \mathrm{C} & -12.51510 \\ \mathrm{O} & -14.60090 \\ \mathrm{H} & -13.09990 \\ \mathrm{H} & -10.65840 \\ \mathrm{H} & -10.56610 \\ \mathrm{H} & -13.06100 \\ \mathrm{C} & -4.22140 \\ \mathrm{H} & -3.33960 \\ \mathrm{H} & -4.22990 \\ \mathrm{H} & -4.20880 \\ \mathrm{C} & -15.39570 \\ \mathrm{H} & -16.43280 \\ \mathrm{H} & -15.22520 \\ \mathrm{H} & -15.20520 \\ & \end{array}$

\section{angstroms}

$\begin{array}{cc}\mathbf{y} & \mathbf{z} \\ -7.61010 & 2.83020 \\ -8.70320 & 2.86250 \\ -8.45810 & 2.87320 \\ -7.13550 & 2.84840 \\ -6.12230 & 2.80840 \\ -9.29090 & 2.90000 \\ -6.52660 & 2.84970 \\ -7.62660 & 2.77750 \\ -6.40490 & 2.77150 \\ -6.39340 & 2.78880 \\ -7.58100 & 2.81130 \\ -8.80250 & 2.81620 \\ -8.82620 & 2.79960 \\ -7.76250 & 2.76230 \\ -5.46450 & 2.75570 \\ -5.43330 & 2.78580 \\ -9.72680 & 2.83280 \\ -9.76400 & 2.80290 \\ -7.40240 & 2.88220 \\ -6.76170 & 2.87870 \\ -8.01690 & 3.79040 \\ -8.05590 & 2.00180 \\ -6.58690 & 2.74160 \\ -6.92520 & 2.73350 \\ -5.96750 & 3.63130 \\ -5.98550 & 1.84370\end{array}$


Total energy............. $\quad-1029.16479807665$ hartrees Number of imaginary frequencies: 0

43:

\begin{tabular}{|c|c|c|c|}
\hline & $\mathrm{X}$ & $y$ & $\mathbf{z}$ \\
\hline C & 4.59160 & 3.41670 & 7.46330 \\
\hline C & 3.85060 & 4.60160 & 7.46520 \\
\hline $\mathrm{C}$ & 2.46340 & 4.55450 & 7.46890 \\
\hline $\mathrm{C}$ & 1.81080 & 3.30500 & 7.47080 \\
\hline C & 2.56170 & 2.11210 & 7.46900 \\
\hline C & 3.94730 & 2.17480 & 7.46530 \\
\hline $\mathrm{H}$ & 5.67600 & 3.45920 & 7.46040 \\
\hline $\mathrm{H}$ & 4.35560 & 5.56140 & 7.46370 \\
\hline $\mathrm{H}$ & 1.88350 & 5.47060 & 7.47030 \\
\hline $\mathrm{H}$ & 2.07810 & 1.13930 & 7.47040 \\
\hline $\mathrm{H}$ & 4.52860 & 1.25940 & 7.46380 \\
\hline $\mathrm{C}$ & 0.37240 & 3.27150 & 7.47460 \\
\hline N & -0.46890 & 2.23250 & 7.47700 \\
\hline $\mathrm{C}$ & -1.78480 & 2.69570 & 7.48040 \\
\hline $\mathrm{C}$ & -1.69310 & 4.03790 & 7.47990 \\
\hline O & -0.35690 & 4.38380 & 7.47640 \\
\hline $\mathrm{H}$ & -2.63600 & 2.03420 & 7.48270 \\
\hline $\mathrm{H}$ & -2.40610 & 4.84520 & 7.48170 \\
\hline $\mathrm{H}$ & -0.19030 & 1.25970 & 7.47640 \\
\hline
\end{tabular}

48:

\section{angstroms}

$\mathrm{X}$

$-4.79170$

$-5.40720$

$-6.71350$

$-7.38050$

$-6.74780$

$-5.41170$

$-4.87960$

$-7.20820$

$-8.39300$

$-7.38990$

$-4.84580$

$-6.80780$

$-8.82900$

$-7.43290$

$-6.69840$

$-5.82350$

$-9.21430$

$-9.06330$

\section{Y}

1.83110

2.81610

2.64060

1. 44860

0.41460

0.65760

3. 73610

3. 42370

1. 35880

$-0.78030$

$-0.07120$

$-1.70600$

$-0.89480$

$-2.61360$

$-1.20920$

$-2.07100$

$-1.90000$

$-0.76550$
1.64300
2.34150
2.78320
2.50040
1.76370
1. 34960
2. 55500
3. 34330
2. 86520
1.48770
0.79000
0.51950
1.70950
0.38210
$-0.46860$
0.88050
1.43680
2.78700 
$\mathrm{H} \quad-9.37220 \quad-0.13960 \quad 1.10140$

Total energy............ $\quad-382.26635394966$ hartrees

Number of imaginary frequencies: 0

49:

angstroms

\section{$\mathbf{x}$}

$\begin{array}{ll}\mathrm{N} & -3.73850 \\ \mathrm{C} & -2.75920 \\ \mathrm{C} & -2.45050 \\ \mathrm{~N} & -3.15930 \\ \mathrm{C} & -4.13010 \\ \mathrm{C} & -4.42660 \\ \mathrm{~N} & -1.46790 \\ \mathrm{H} & -4.69180 \\ \mathrm{H} & -5.21720 \\ \mathrm{C} & -2.01270 \\ \mathrm{~N} & -0.96890 \\ \mathrm{~N} & -2.29450 \\ \mathrm{C} & -1.34640 \\ \mathrm{C} & -0.53240 \\ \mathrm{C} & 0.52360 \\ \mathrm{~N} & 0.77410 \\ \mathrm{C} & -0.01920 \\ \mathrm{C} & -1.09610 \\ \mathrm{H} & 1.17050 \\ \mathrm{H} & 0.23140 \\ \mathrm{H} & -1.68500 \\ \mathrm{C} & -3.34490 \\ \mathrm{C} & -2.93170 \\ \mathrm{H} & -3.72590 \\ \mathrm{H} & -2.77030 \\ \mathrm{H} & -2.01500 \\ \mathrm{H} & -4.23650 \\ \mathrm{H} & -3.56100 \\ \mathrm{H} & -1.31270 \\ \mathrm{H} & -0.89180 \\ \mathrm{H} & -1.20\end{array}$

\section{$\mathbf{y}$}

2.63930

2.62260

1.40150

0.27970

0.34690

1.51940

1.31540

$-0.56760$

1.55940

3.85860

3.90980

5.10230

5.97360

5.21460

5.85000

7.14680

7.85170

7.33460

5.29550

8.90520

7.96340

5.50810

5.32770

5.69610

4.26960

5.88050

4.92390

6.55880

0.41850

2.13490
3.07350

2.16460

1. 46050

1.70320

2.60990

3.30410

0.54380

2.79220

4.04780

1. 91220

1.09280

2.47950

1.98310

1.11740

0.45280

0.62280

1. 45950

2.17350

$-0.22420$

1. 56350

2.83250

3.42420

4.88380

5.54130

5.10200

5.11040

3.20880

3.20880

0.11180

0.36880

Total energy............ $\quad-793.05981281735$ hartrees

Number of imaginary frequencies: 0

119:

\section{angstroms}

\section{$\mathbf{X}$}

$\begin{array}{ll}\mathrm{C} & 0.98710 \\ \mathrm{~N} & 1.65330 \\ \mathrm{C} & 2.93950 \\ \mathrm{C} & 2.98750 \\ \mathrm{O} & 1.76840\end{array}$

\section{$y$}

$-13.13060$

$-14.30430$

$-13.93650$

$-12.56180$

$-12.06290$
3.13070
3.08280
3.13790
3.21600
3.21120 


\begin{tabular}{|c|c|c|c|}
\hline $\mathrm{H}$ & 3.87360 & -11.94580 & 3.27430 \\
\hline $\mathrm{C}$ & -3.22160 & -12.85980 & 3.04450 \\
\hline $\mathrm{C}$ & -2.44600 & -11.70120 & 3.12570 \\
\hline C & -1.05080 & -11.79010 & 3.15360 \\
\hline $\mathrm{C}$ & -0.41320 & -13.04540 & 3.10020 \\
\hline $\mathrm{C}$ & -1.20840 & -14.20700 & 3.01770 \\
\hline $\mathrm{C}$ & -2.60310 & -14.11080 & 2.99080 \\
\hline $\mathrm{F}$ & -4.57420 & -12.77010 & 3.01840 \\
\hline $\mathrm{H}$ & -2.92530 & -10.73150 & 3.16800 \\
\hline $\mathrm{H}$ & -0.47470 & -10.87500 & 3.21730 \\
\hline $\mathrm{H}$ & -0.75720 & -15.19080 & 2.97500 \\
\hline $\mathrm{H}$ & -3.20430 & -15.00890 & 2.92840 \\
\hline C & 6.16400 & -16.65090 & 3.09120 \\
\hline $\mathrm{C}$ & 4.84650 & -17.12720 & 3.01790 \\
\hline C & 3.78200 & -16.22560 & 3.03230 \\
\hline $\mathrm{C}$ & 4.01820 & -14.84140 & 3.11900 \\
\hline C & 5.35610 & -14.40680 & 3.18640 \\
\hline $\mathrm{N}$ & 6.37390 & -15.30710 & 3.17150 \\
\hline $\mathrm{H}$ & 4.64410 & -18.18850 & 2.94690 \\
\hline $\mathrm{H}$ & 2.77370 & -16.61740 & 2.97530 \\
\hline $\mathrm{H}$ & 5.60630 & -13.35830 & 3.25330 \\
\hline $\mathrm{C}$ & 7.33650 & -17.62130 & 3.08440 \\
\hline O & 7.18980 & -18.48040 & 1.97770 \\
\hline $\mathrm{C}$ & 7.32310 & -18.44040 & 4.39090 \\
\hline $\mathrm{H}$ & 8.19800 & -19.12430 & 4.43310 \\
\hline $\mathrm{H}$ & 7.36220 & -17.76620 & 5.27390 \\
\hline $\mathrm{H}$ & 6.40340 & -19.05940 & 4.45720 \\
\hline $\mathrm{C}$ & 8.68150 & -16.87200 & 2.96530 \\
\hline $\mathrm{H}$ & 9.52870 & -17.59070 & 2.94740 \\
\hline $\mathrm{H}$ & 8.71080 & -16.28210 & 2.02400 \\
\hline $\mathrm{H}$ & 8.82960 & -16.18430 & 3.82610 \\
\hline $\mathrm{H}$ & 7.92770 & -19.14540 & 2.0028 \\
\hline
\end{tabular}

Total energy............. -1016.65603321885 hartrees Number of imaginary frequencies: 0

120:

angstroms

$\mathbf{x}$

$\begin{array}{lr}\mathrm{C} & 0.00570 \\ \mathrm{C} & 1.34300 \\ \mathrm{C} & 2.19120 \\ \mathrm{C} & 1.49770 \\ \mathrm{~S} & -0.21920 \\ \mathrm{H} & 1.68600 \\ \mathrm{H} & 3.27400 \\ \mathrm{H} & 1.88330 \\ \mathrm{C} & -1.10630 \\ \mathrm{~N} & -0.87170 \\ \mathrm{C} & -2.06510 \\ \mathrm{O} & -3.08730\end{array}$

\section{$\mathrm{Y}$}

$-0.40280$

$-0.08880$

$-1.23380$

$-2.41060$

$-2.14010$

0.93770

$-1.18250$

$-3.42010$

0.52920

1. 91210

2.42740

1.53460
1.93550

1.96010

1.93840

1.89730

1.88430

1.99290

1.95280

1.87390

1.94840

1.98930

1.98990

1.95350 


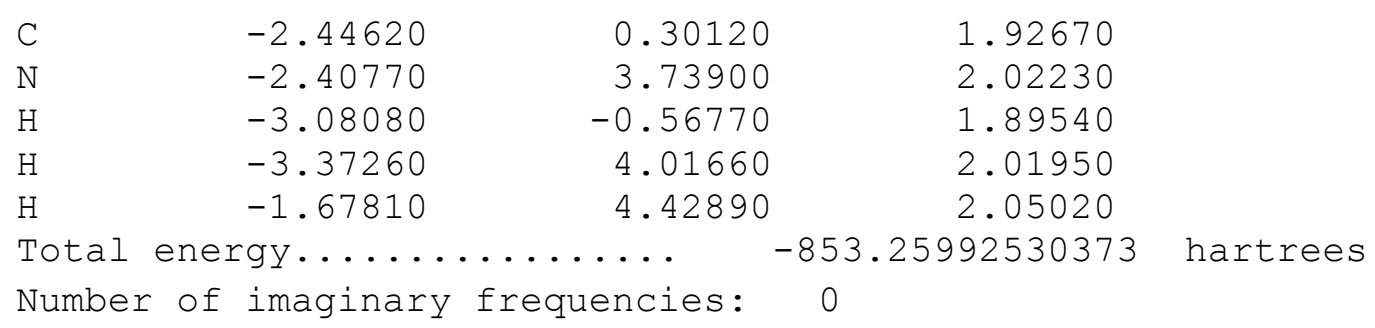

121:

angstroms

$\mathrm{X}$

$\begin{array}{ll}\mathrm{C} & -8.43350 \\ \mathrm{~N} & -7.59880 \\ \mathrm{C} & -6.39990 \\ \mathrm{O} & -6.32830 \\ \mathrm{C} & -7.65690 \\ \mathrm{H} & -7.82530 \\ \mathrm{~N} & -5.22090 \\ \mathrm{H} & -4.34890 \\ \mathrm{H} & -5.24000 \\ \mathrm{C} & -12.73580 \\ \mathrm{C} & -12.04780 \\ \mathrm{C} & -10.64700 \\ \mathrm{C} & -9.90280 \\ \mathrm{C} & -10.63310 \\ \mathrm{C} & -12.02060 \\ \mathrm{H} & -13.82110 \\ \mathrm{H} & -12.59560 \\ \mathrm{O} & -9.99600 \\ \mathrm{H} & -10.08880 \\ \mathrm{H} & -12.53770 \\ \mathrm{H} & -10.65900 \\ \mathrm{~T} & -10.62 . . .\end{array}$

\section{$y$}

$-7.79120$

$-8.91730$

$-8.42310$

$-7.06910$

$-6.67190$

$-5.60980$

$-9.09590$

$-8.60140$

$-10.09910$

$-7.79740$

$-9.00430$

$-9.04590$

$-7.84590$

$-6.64260$

$-6.60190$

$-7.79290$

$-9.94340$

$-10.24210$

$-5.70520$

$-5.64780$

$-10.94350$

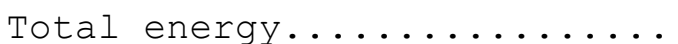

Number of imaginary frequencies:

7.67030

7.66620

7.72700

7.77220

7.73470

7.76150

7.75020

7.79870

7.71780

7.51060

7.48850

7.53880

7.61390

7.63440

7.58430

7.47040

7.43100

7.51530

7.69220

7.60290

7.46240

-607.71825554386 hartrees

122:

angstroms

$\mathrm{X}$

$\begin{array}{lr}\mathrm{C} & 13.66800 \\ \mathrm{C} & 10.83120 \\ \mathrm{C} & 11.63820 \\ \mathrm{C} & 13.04260 \\ \mathrm{H} & 14.75080 \\ \mathrm{H} & 9.75160 \\ \mathrm{H} & 11.18070 \\ \mathrm{H} & 13.64660 \\ \mathrm{C} & 12.04960 \\ \mathrm{C} & 10.93980 \\ \mathrm{C} & 11.43230\end{array}$

\section{$y$}

$-13.12390$

$-13.33860$

$-14.46890$

$-14.36380$

$-13.03760$

$-13.44370$

$-15.45360$

$-15.26610$

$-9.88730$

$-10.70600$

$-12.06810$
5.00870
5.00550
5.00030
5.00130
5.00800
5.00430
4.99420
4.99520
5.02150
5.01590
5.01300 


\begin{tabular}{lrrr}
$\mathrm{C}$ & 12.85120 & -11.99040 & 5.01570 \\
$\mathrm{~N}$ & 13.19170 & -10.65160 & 5.02110 \\
$\mathrm{H}$ & 12.09860 & -8.80930 & 5.02820 \\
$\mathrm{H}$ & 14.13050 & -10.28850 & 5.02370 \\
$\mathrm{C}$ & 9.57090 & -10.25000 & 5.01690 \\
$\mathrm{C}$ & 8.33780 & -10.83910 & 4.99150 \\
$\mathrm{~N}$ & 7.34680 & -9.86500 & 5.00370 \\
$\mathrm{C}$ & 7.99990 & -8.75050 & 5.03410 \\
$\mathrm{O}$ & 9.35390 & -8.88570 & 5.04370 \\
$\mathrm{H}$ & 8.09130 & -11.88880 & 4.96700 \\
$\mathrm{H}$ & 7.61320 & -7.74270 & 5.05320 \\
Total energy.............. & -608.71835907225 & hartrees \\
\multicolumn{2}{l}{ Number of imaginary frequencies: } & 0 &
\end{tabular}

123:

angstroms

\section{$\mathbf{x}$ \\ $y$}

\begin{tabular}{lr}
$\mathrm{C}$ & 1.05710 \\
$\mathrm{~N}$ & 1.67130 \\
$\mathrm{C}$ & 3.00450 \\
$\mathrm{C}$ & 3.13270 \\
$\mathrm{O}$ & 1.89660 \\
$\mathrm{H}$ & 3.96000 \\
$\mathrm{C}$ & -3.06770 \\
$\mathrm{C}$ & -2.22670 \\
$\mathrm{C}$ & -0.86840 \\
$\mathrm{C}$ & -0.36150 \\
$\mathrm{C}$ & -1.23700 \\
$\mathrm{C}$ & -2.59470 \\
$\mathrm{~F}$ & -4.38290 \\
$\mathrm{H}$ & -2.63930 \\
$\mathrm{H}$ & -0.19690 \\
$\mathrm{H}$ & -0.83790 \\
$\mathrm{H}$ & -3.28740 \\
$\mathrm{C}$ & 6.01970 \\
$\mathrm{C}$ & 4.75890 \\
$\mathrm{C}$ & 3.72750 \\
$\mathrm{C}$ & 4.03810 \\
$\mathrm{~N}$ & 5.28140 \\
$\mathrm{~N}$ & 6.27290 \\
$\mathrm{H}$ & 4.62560 \\
$\mathrm{H}$ & 2.70580 \\
$\mathrm{~S}$ & 7.45540 \\
$\mathrm{O}$ & 8.29650 \\
$\mathrm{C}$ & 8.34380 \\
$\mathrm{O}$ & 6.91180 \\
$\mathrm{H}$ & 7.73560 \\
$\mathrm{H}$ & 9.26670 \\
$\mathrm{H}$ & 8.55080 \\
& \\
\hline
\end{tabular}

$-13.20210$

$-14.33320$

$-14.07570$

$-12.76910$

$-12.19860$

$-12.14950$

$-12.38620$

$-11.35610$

$-11.61910$

$-12.90190$

$-13.92170$

$-13.66800$

$-12.13590$

$-10.37260$

$-10.82850$

$-14.91050$

$-14.44080$

$-16.84610$

$-17.32140$

$-16.40530$

$-15.10310$

$-14.72750$

$-15.60680$

$-18.35490$

$-16.65430$

$-17.95470$

$-17.51580$

$-17.63870$

$-19.32270$

$-17.97910$

$-18.21720$

$-16.57090$
3.22670

3.02850

3. 32190

3.69040

3.63480

3.99090

2. 78240

3.18810

3.33410

3.07570

2.66640

2.51780

2.64100

3. 38310

3.65000

2. 46860

2. 20370

3.10920

2. 74060

2. 79380

3.22550

3.58530

3.51540

2. 44150

2. 53050

2. 99290

1.87240

4.52700

3.02460

5.36580

4.45850

4.59080 
Total energy............. $\quad-1427.37508329945$ hartrees Number of imaginary frequencies: 0

$124:$

\section{angstroms}

\section{$\mathbf{X}$}

$\begin{array}{lr}\text { N } & 0.91450 \\ \mathrm{C} & 0.37960 \\ \mathrm{C} & -1.00510 \\ \mathrm{C} & -1.84740 \\ \mathrm{C} & -1.28830 \\ \mathrm{C} & 0.14310 \\ \mathrm{H} & 1.03880 \\ \mathrm{~F} & -1.53070 \\ \mathrm{H} & -2.91600 \\ \mathrm{~N} & -2.10000 \\ \mathrm{C} & -3.53850 \\ \mathrm{C} & -4.14050 \\ \mathrm{~N} & -4.87350 \\ \mathrm{O} & -5.28870 \\ \mathrm{C} & -4.77990 \\ \mathrm{C} & -4.03930 \\ \mathrm{H} & -5.03220 \\ \mathrm{H} & -3.50830 \\ \mathrm{H} & -1.64040 \\ \mathrm{C} & 0.94530 \\ \mathrm{O} & 2.13120 \\ \mathrm{O} & 0.20090 \\ \mathrm{C} & 0.93400 \\ \mathrm{C} & -0.03850 \\ \mathrm{H} & -3.81350 \\ \mathrm{H} & -3.98270 \\ \mathrm{H} & 1.37620 \\ \mathrm{H} & 1.75510 \\ \mathrm{H} & 0.48210 \\ \mathrm{H} & -0.86030 \\ \mathrm{H} & -0.46200 \\ \mathrm{H} & -120\end{array}$

\section{Y}

1.92980

3.14130

3.29940

2.20920

0.91220

0.83520

4.00400

4.53680

2. 37160

$-0.19120$

$-0.12080$

$-1.49210$

$-1.95770$

$-3.22650$

$-3.48210$

$-2.42940$

$-4.44260$

$-2.32020$

$-1.06740$

$-0.43230$

$-0.49750$

$-1.54820$

$-2.79780$

$-3.90320$

0.30600

0.52020

$-2.94220$

$-2.71900$

$-4.86580$

$-3.96060$

$-3.74430$
2.45750

2.45090

2.39660

2. 35020

2. 36380

2.41150

2.48510

2. 38180

2.29730

2.36090

2.16510

2.27050

1.28300

1.68960

2.90860

3.34360

3.33390

4.27610

2.15440

2.42930

2.65200

2.14850

2.14860

1.78640

1.18980

2.93880

3.13870

1.43080

1.78600

2.50690

0.79030

Total energy............. $\quad-954.23169409503$ hartrees

Number of imaginary frequencies: 0

125:

$\mathrm{x}$

$\begin{array}{ll}\mathrm{C} & -1.30380 \\ \mathrm{~N} & -1.93930 \\ \mathrm{C} & -3.26520 \\ \mathrm{C} & -4.03890 \\ \mathrm{C} & -3.34180\end{array}$

\section{angstroms}

\section{Y}

3.33330

4.42000

4.34570

3.21360

2.08840
1.72860

2.18310

2.32510

2.00860

1.53740 


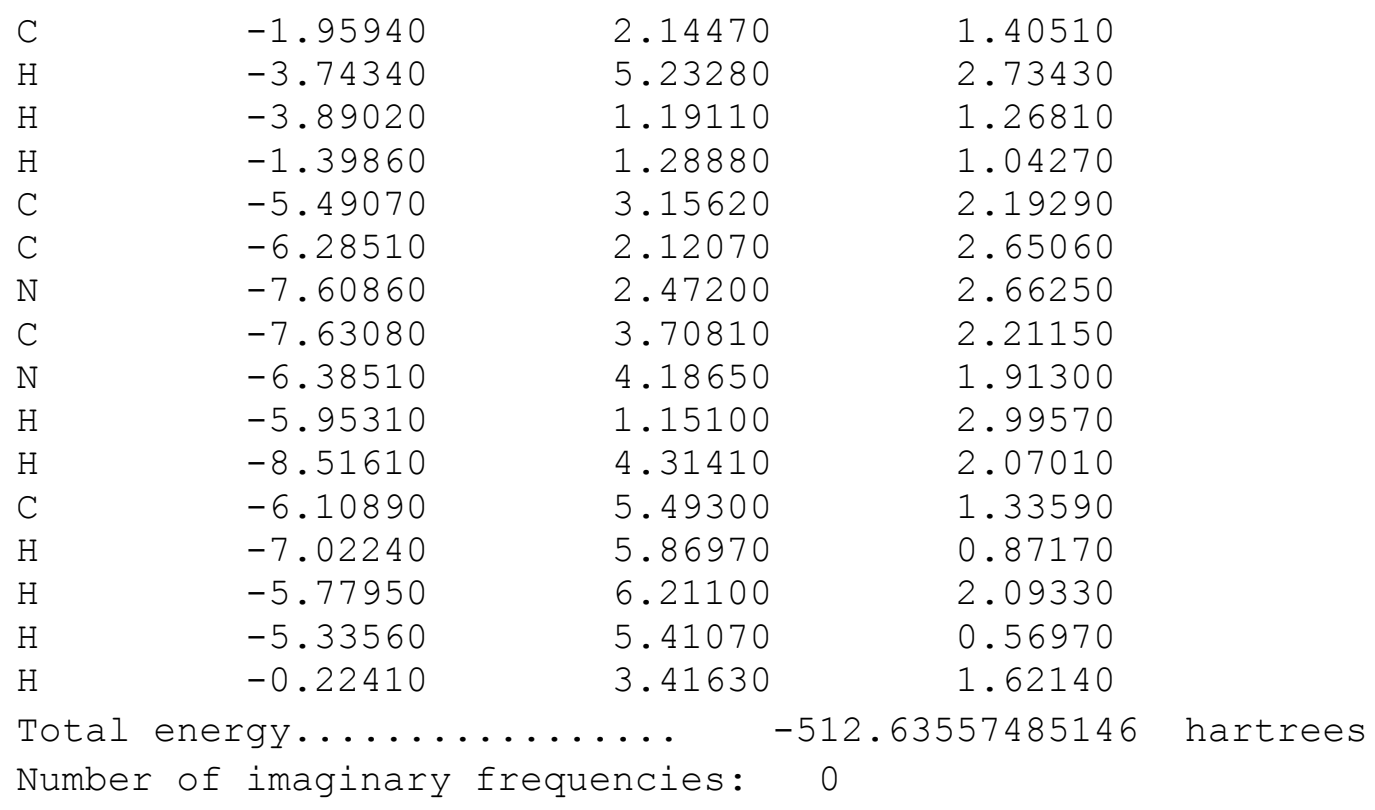

126:

angstroms

$\begin{array}{lrcc} & \mathbf{X} & \mathbf{y} & \mathbf{z} \\ \mathrm{C} & -1.20810 & 3.22390 & 1.86550 \\ \mathrm{C} & -1.87260 & 4.35310 & 2.35510 \\ \mathrm{C} & -3.26590 & 4.35020 & 2.44970 \\ \mathrm{C} & -4.02970 & 3.24460 & 2.05020 \\ \mathrm{C} & -3.33620 & 2.11950 & 1.55900 \\ \mathrm{C} & -1.95350 & 2.10260 & 1.47360 \\ \mathrm{O} & 0.14530 & 3.11180 & 1.73010 \\ \mathrm{H} & -1.32210 & 5.22840 & 2.67850 \\ \mathrm{H} & -3.75650 & 5.22130 & 2.87240 \\ \mathrm{H} & -3.90330 & 1.25460 & 1.22920 \\ \mathrm{H} & -1.42430 & 1.23550 & 1.09240 \\ \mathrm{C} & -5.49210 & 3.19220 & 2.16500 \\ \mathrm{C} & -6.31640 & 2.15770 & 2.56640 \\ \mathrm{~N} & -7.63920 & 2.51540 & 2.50800 \\ \mathrm{C} & -7.63080 & 3.75570 & 2.07140 \\ \mathrm{~N} & -6.36610 & 4.22960 & 1.84720 \\ \mathrm{H} & -6.00850 & 1.18170 & 2.91550 \\ \mathrm{H} & -8.50330 & 4.36980 & 1.89040 \\ \mathrm{C} & 0.95270 & 4.21330 & 2.11500 \\ \mathrm{H} & 1.98360 & 3.91280 & 1.92270 \\ \mathrm{H} & 0.72250 & 5.11020 & 1.52540 \\ \mathrm{H} & 0.83810 & 4.44680 & 3.18130 \\ \mathrm{C} & -6.04960 & 5.53700 & 1.29790 \\ \mathrm{H} & -6.93060 & 5.92280 & 0.78080 \\ \mathrm{H} & -5.76370 & 6.25010 & 2.07820 \\ \mathrm{H} & -5.22960 & 5.45460 & 0.58170 \\ \mathrm{H} & -50100\end{array}$

Total energy............. $\quad-611.12583785756$ hartrees Number of imaginary frequencies: 0 


\begin{tabular}{|c|c|c|c|}
\hline & & & \\
\hline & $\mathbf{x}$ & $y$ & $\mathbf{z}$ \\
\hline C & 0.93540 & 0.61530 & 0.73210 \\
\hline $\mathrm{C}$ & 1.01090 & 1.90910 & 1.25870 \\
\hline $\mathrm{C}$ & 0.26100 & 2.23540 & 2.39790 \\
\hline C & -0.55030 & 1.28290 & 2.99590 \\
\hline $\mathrm{C}$ & -0.65880 & -0.02110 & 2.47170 \\
\hline C & 0.10160 & -0.32860 & 1.33490 \\
\hline $\mathrm{H}$ & 1.50650 & 0.33530 & -0.14510 \\
\hline O & 1.77700 & 2.91600 & 0.74860 \\
\hline $\mathrm{H}$ & 0.34470 & 3.23830 & 2.80360 \\
\hline $\mathrm{H}$ & -1.08840 & 1.54230 & 3.90320 \\
\hline $\mathrm{H}$ & 0.03380 & -1.32530 & 0.91050 \\
\hline C & -1.48980 & -1.05590 & 3.10440 \\
\hline C & -1.17060 & -2.34320 & 3.48610 \\
\hline $\mathrm{N}$ & -2.24060 & -2.97490 & 4.07090 \\
\hline C & -3.20950 & -2.08690 & 4.04280 \\
\hline $\mathrm{N}$ & -2.82290 & -0.89750 & 3.48510 \\
\hline $\mathrm{H}$ & -0.20580 & -2.82410 & 3.39980 \\
\hline $\mathrm{H}$ & -4.21810 & -2.24030 & 4.40290 \\
\hline C & -3.74150 & 0.19290 & 3.14370 \\
\hline $\mathrm{H}$ & -3.13850 & 1.07840 & 2.93590 \\
\hline $\mathrm{H}$ & -4.35000 & 0.41370 & 4.02630 \\
\hline C & -6.28920 & -0.71900 & -0.22770 \\
\hline $\mathrm{C}$ & -6.84480 & -0.53020 & 1.03620 \\
\hline C & -6.02080 & -0.23480 & 2.12390 \\
\hline C & -4.63730 & -0.12440 & 1.96020 \\
\hline C & -4.08750 & -0.31030 & 0.68500 \\
\hline C & -4.90770 & -0.60760 & -0.40040 \\
\hline $\mathrm{H}$ & -6.92740 & -0.95230 & -1.07510 \\
\hline $\mathrm{H}$ & -7.91820 & -0.61570 & 1.17880 \\
\hline $\mathrm{H}$ & -6.45910 & -0.09080 & 3.10880 \\
\hline $\mathrm{H}$ & -3.01320 & -0.22580 & 0.54610 \\
\hline $\mathrm{H}$ & -4.46940 & -0.75190 & -1.38380 \\
\hline $\mathrm{C}$ & 2.57000 & 2.64470 & -0.39670 \\
\hline $\mathrm{H}$ & 3.09260 & 3.57420 & -0.62740 \\
\hline $\mathrm{H}$ & 3.30690 & 1.85530 & -0.20130 \\
\hline $\mathrm{H}$ & 1.95340 & 2.35490 & -1.25710 \\
\hline
\end{tabular}

Total energy............. $\quad-842.18149914516$ hartrees Number of imaginary frequencies: 0

128:

\begin{tabular}{cccc} 
& \multicolumn{3}{c}{ angstroms } \\
$\mathrm{N}$ & $\mathbf{X}$ & $\mathbf{y}$ & $\mathbf{z}$ \\
$\mathrm{C}$ & 0.52420 & -0.02130 & -4.39780 \\
$\mathrm{C}$ & -0.07070 & 1.16020 & -4.49020 \\
$\mathrm{C}$ & -1.46210 & 1.28980 & -4.56850 \\
& -2.24150 & 0.14830 & -4.54210
\end{tabular}




\begin{tabular}{|c|c|c|c|}
\hline $\mathrm{C}$ & -1.63850 & -1.12650 & -4.42450 \\
\hline $\mathrm{C}$ & -0.20900 & -1.14420 & -4.36640 \\
\hline $\mathrm{H}$ & 0.57700 & 2.03380 & -4.51070 \\
\hline $\mathrm{H}$ & -1.92250 & 2.26910 & -4.65870 \\
\hline $\mathrm{H}$ & -3.31930 & 0.22960 & -4.62280 \\
\hline $\mathrm{N}$ & -2.41180 & -2.25780 & -4.35310 \\
\hline $\mathrm{C}$ & 0.64420 & -2.37580 & -4.26270 \\
\hline O & 1.84030 & -2.37600 & -4.09030 \\
\hline O & -0.06300 & -3.54360 & -4.39470 \\
\hline $\mathrm{C}$ & 0.72530 & -4.75560 & -4.30760 \\
\hline $\mathrm{C}$ & -0.22060 & -5.93330 & -4.44020 \\
\hline $\mathrm{H}$ & 1.25800 & -4.75830 & -3.35240 \\
\hline $\mathrm{H}$ & 1.47700 & -4.73930 & -5.10210 \\
\hline $\mathrm{H}$ & 0.34460 & -6.86880 & -4.38520 \\
\hline $\mathrm{H}$ & -0.96100 & -5.93810 & -3.63420 \\
\hline $\mathrm{H}$ & -0.74740 & -5.91090 & -5.39900 \\
\hline $\mathrm{H}$ & -1.91830 & -3.13540 & -4.43530 \\
\hline C & -3.84520 & -2.27000 & -4.42440 \\
\hline C & -4.51270 & -3.64850 & -4.25340 \\
\hline O & -5.32170 & -3.45190 & -5.43110 \\
\hline $\mathrm{C}$ & -4.57270 & -2.27060 & -5.80000 \\
\hline $\mathrm{H}$ & -4.26450 & -1.52340 & -3.74210 \\
\hline $\mathrm{H}$ & -3.81280 & -4.49080 & -4.36680 \\
\hline $\mathrm{H}$ & -5.11480 & -3.80550 & -3.35190 \\
\hline $\mathrm{H}$ & -3.89770 & -2.45120 & -6.64740 \\
\hline $\mathrm{H}$ & -5.22970 & -1.42280 & -6.02690 \\
\hline
\end{tabular}

129:

\section{angstroms}

\section{$\mathbf{x}$}

$\begin{array}{ll}\mathrm{N} & -4.72660 \\ \mathrm{C} & -5.50030 \\ \mathrm{C} & -6.88990 \\ \mathrm{C} & -7.49950 \\ \mathrm{C} & -6.70910 \\ \mathrm{C} & -5.31710 \\ \mathrm{H} & -4.99340 \\ \mathrm{H} & -7.48640 \\ \mathrm{H} & -8.58070 \\ \mathrm{H} & -4.64320 \\ \mathrm{C} & -7.30380 \\ \mathrm{C} & -6.44120 \\ \mathrm{~N} & -7.29970 \\ \mathrm{C} & -8.31870 \\ \mathrm{C} & -9.11110 \\ \mathrm{O} & -8.25510 \\ \mathrm{H} & -6.67820 \\ \mathrm{H} & -5.80860\end{array}$

$\mathbf{y}$
1.74200
2.82790
2.76940
1.53800
0.37660
0.55930
3.77780
3.67410
1.47260
-0.28970
-3.31950
-2.07140
-0.89130
-1.04720
-2.33070
-3.46320
-4.21630
-2.19350

2. 34220

2. 41230

2. 29100

2.09470

2.01290

2.14120

2.56860

2.36200

2.03070

2.07650

2.13800

1.95300

1.85860

0.81100

1.04230

1.09300

2.11900

1.05590 


\begin{tabular}{|c|c|c|c|}
\hline $\mathrm{H}$ & -5.78210 & -1.96860 & 2.82010 \\
\hline $\mathrm{H}$ & -9.00270 & -0.19630 & 0.82430 \\
\hline $\mathrm{H}$ & -7.84860 & -1.08460 & -0.18710 \\
\hline $\mathrm{H}$ & -9.68470 & -2.24380 & 1.97960 \\
\hline $\mathrm{H}$ & -9.80880 & -2.50020 & 0.21730 \\
\hline $\mathrm{H}$ & -7.81510 & -3.26610 & 3.11280 \\
\hline
\end{tabular}

130:

\section{angstroms}

$\begin{array}{lccc} & \mathbf{X} & \mathbf{y} & \mathbf{z} \\ \mathrm{N} & -4.73130 & 1.74330 & 2.16870 \\ \mathrm{C} & -5.51620 & 2.81310 & 2.33790 \\ \mathrm{C} & -6.91050 & 2.73590 & 2.36290 \\ \mathrm{C} & -7.52410 & 1.49760 & 2.20610 \\ \mathrm{C} & -6.71040 & 0.37690 & 2.02910 \\ \mathrm{C} & -5.32160 & 0.55580 & 2.01830 \\ \mathrm{H} & -5.01140 & 3.76900 & 2.45820 \\ \mathrm{H} & -7.50670 & 3.63230 & 2.50300 \\ \mathrm{H} & -8.60280 & 1.38210 & 2.21770 \\ \mathrm{H} & -4.66380 & -0.30500 & 1.88150 \\ \text { O } & -7.30360 & -0.84100 & 1.87460 \\ \mathrm{H} & -6.61880 & -1.51460 & 1.76300 \\ \text { Total energy............. } & -323.51235623991 & \text { hartrees } \\ \text { Number of } & \text { imaginary } & \text { frequencies: } & \end{array}$

\section{1:}

$\mathrm{X}$

$\begin{array}{ll}\mathrm{N} & 2.88970 \\ \mathrm{C} & 2.24990 \\ \mathrm{C} & 0.83600 \\ \mathrm{~N} & 0.09320 \\ \mathrm{C} & 0.72880 \\ \mathrm{C} & 2.13070 \\ \mathrm{~N} & 3.02070 \\ \mathrm{H} & 0.31950 \\ \mathrm{H} & 0.11250 \\ \mathrm{C} & 2.81020 \\ \mathrm{O} & 4.17830 \\ \mathrm{C} & 4.58190 \\ \mathrm{C} & 3.50880 \\ \mathrm{C} & 2.35570 \\ \mathrm{H} & 5.64860 \\ \mathrm{H} & 3.53530 \\ \mathrm{H} & 1.32380 \\ \mathrm{H} & 2.61550 \\ \mathrm{H} & 4.02140\end{array}$

\section{angstroms}

$\begin{array}{rc}\mathbf{y} & \mathbf{z} \\ 2.29240 & 2.91970 \\ 3.46740 & 2.92710 \\ 3.53390 & 2.96190 \\ 2.43460 & 2.98850 \\ 1.25840 & 2.98130 \\ 1.18340 & 2.94680 \\ 4.59340 & 2.89960 \\ 4.49230 & 2.96780 \\ 0.36490 & 3.00330 \\ -0.10720 & 2.93950 \\ -0.10610 & 2.90610 \\ -1.40210 & 2.90560 \\ -2.24250 & 2.93780 \\ -1.40320 & 2.95990 \\ -1.56170 & 2.88070 \\ -3.32230 & 2.94490 \\ -1.72080 & 2.98730 \\ 5.51250 & 2.90310 \\ 4.48870 & 2.87540\end{array}$


Total energy............. $\quad-548.53058811159$ hartrees Number of imaginary frequencies: 0

132:

\section{angstroms}

$\mathbf{x}$

$\mathrm{N} \quad 2.90070$

C $\quad 2.27160$

C $\quad 0.85960$

$\mathrm{N} \quad 0.13200$

C $\quad 0.74480$

C $\quad 2.16000$

$\mathrm{N} \quad 3.03890$

$\mathrm{H} \quad 0.33570$

C $\quad 2.92390$

O 4.23300

C $\quad 4.78130$

C $\quad 3.86500$

C $\quad 2.65820$

$\mathrm{H} \quad 5.81630$

$\mathrm{H} \quad 4.02600$

$\mathrm{H} \quad 1.71680$

$\mathrm{H} \quad 2.63150$

$\mathrm{H} \quad 4.03940$

$\mathrm{N} \quad-2.03250$

C $\quad-2.26430$

C $\quad-1.38630$

C $\quad-0.17200$

$C$
$C$$\quad 0.07090$

C $\quad-0.87870$

$\mathrm{H} \quad-3.20680$

$\mathrm{H} \quad-1.63690$

$\mathrm{H} \quad 0.97770$

$\mathrm{H} \quad-0.70500$

$\begin{array}{rc}\mathbf{y} & \mathbf{z} \\ 2.17170 & 3.01030 \\ 3.34650 & 3.06230 \\ 3.40720 & 2.97710 \\ 2.30640 & 2.97710 \\ 1.10530 & 3.02560 \\ 1.04980 & 2.96310 \\ 4.46770 & 3.17000 \\ 4.36040 & 2.92180 \\ -0.18460 & 2.76190 \\ -0.16690 & 3.17080 \\ -1.35650 & 2.81960 \\ -2.14180 & 2.18540 \\ -1.38250 & 2.14690 \\ -1.49500 & 3.09090 \\ -3.13520 & 1.79290 \\ -1.67990 & 1.71180 \\ 5.38420 & 3.22570 \\ 4.36410 & 3.20830 \\ -2.16830 & 3.48040 \\ -1.14210 & 2.65130 \\ -0.07740 & 2.46360 \\ -0.05730 & 3.16350 \\ -1.12270 & 4.04240 \\ -2.13510 & 4.16040 \\ -1.17080 & 2.10670 \\ 0.73650 & 1.79240 \\ -1.16080 & 4.63480 \\ -2.95670 & 4.83960 \\ -1 . .0 & 2900\end{array}$

Total energy............ $\quad-795.62429293646$

Number of imaginary frequencies: 0

133:

angstroms

$\mathrm{X}$

$\begin{array}{lr}\mathrm{C} & -7.30960 \\ \mathrm{C} & -8.02150 \\ \mathrm{~N} & -9.33960 \\ \mathrm{C} & -10.02090 \\ \mathrm{C} & -9.31010 \\ \mathrm{~N} & -7.96580 \\ \mathrm{~N} & -5.95120 \\ \mathrm{H} & -7.50020 \\ \mathrm{~N} & -14.28260\end{array}$

\section{$\mathbf{Y}$}

4.10060

5.32450

5.34530

4.17860

2.95970

2.94030

4.05430

6.28010

4.67830
4.93480

4.89480

4.91150

4.93120

4.82680

4.86080

5.02140

4.85910

5.40370 


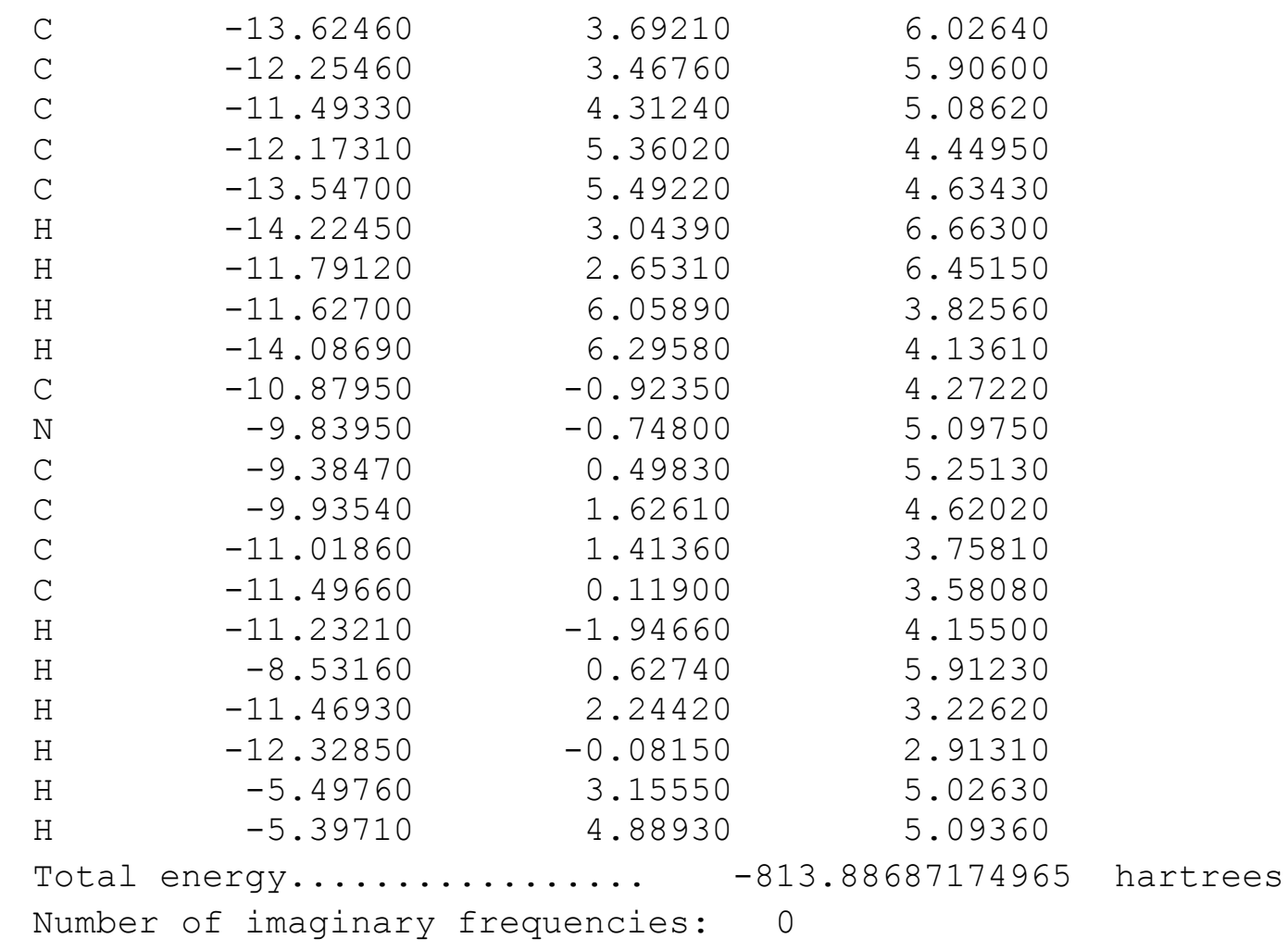

134:

angstroms

\section{$\mathrm{x}$}

$\begin{array}{ll}\mathrm{N} & -2.84640 \\ \mathrm{C} & -3.64870 \\ \mathrm{C} & -5.04300 \\ \mathrm{~N} & -5.63950 \\ \mathrm{C} & -4.86270 \\ \mathrm{C} & -3.43150 \\ \mathrm{H} & -3.16580 \\ \mathrm{H} & -5.67910 \\ \mathrm{~N} & -2.61810 \\ \mathrm{C} & -5.51730 \\ \mathrm{~N} & -4.93700 \\ \mathrm{O} & -6.88730 \\ \mathrm{C} & -5.98500 \\ \mathrm{C} & -7.19090 \\ \mathrm{C} & -8.44010 \\ \mathrm{C} & -8.44060 \\ \mathrm{C} & -7.24400 \\ \mathrm{C} & -5.99870 \\ \mathrm{H} & -9.35480 \\ \mathrm{H} & -9.38890 \\ \mathrm{H} & -7.29580 \\ \mathrm{H} & -5.07440 \\ \mathrm{H} & -3.02110\end{array}$

\section{$\mathrm{Y}$}

$-3.08020$

$-2.01990$

$-2.12620$

$-3.31680$

$-4.40440$

$-4.29530$

$-1.04420$

$-1.24570$

$-5.37130$

$-5.70380$

$-6.87700$

$-5.74010$

$-7.79110$

$-7.07730$

$-7.67620$

$-9.07270$

$-9.81230$

$-9.18800$

$-7.09500$

$-9.60060$

$-10.89670$

$-9.75540$

$-6.30180$
3.86480

3.87980

3.90560

3. 91560

3.90050

3.87500

3.87120

3.91830

3.86090

3.91180

3.90010

3.93980

3.92160

3.94600

3.97140

3.97160

3.94750

3.92200

3.98970

3.99090

3.94870

3.90330

3.86630 
$\mathrm{H} \quad-1.62450 \quad-5.20660 \quad 3.84130$

Total energy............ $\quad-718.24454755747$ hartrees

Number of imaginary frequencies: 0

135:

\begin{tabular}{|c|c|c|c|}
\hline $\mathrm{N}$ & 3.22560 & 2.40530 & 2.78040 \\
\hline C & 2.43650 & 3.48260 & 2.75590 \\
\hline C & 1.04180 & 3.41340 & 2.68190 \\
\hline $\mathrm{N}$ & 0.43750 & 2.20110 & 2.66410 \\
\hline C & 1.20150 & 1.12790 & 2.68710 \\
\hline C & 2.62000 & 1.21070 & 2.72840 \\
\hline $\mathrm{N}$ & 3.38880 & 0.07830 & 2.69670 \\
\hline C & -1.44250 & 6.75260 & 2.65930 \\
\hline $\mathrm{N}$ & -1.99850 & 5.58510 & 3.00230 \\
\hline $\mathrm{C}$ & -1.18600 & 4.52590 & 2.99880 \\
\hline $\mathrm{C}$ & 0.17390 & 4.60610 & 2.65830 \\
\hline C & 0.61890 & 5.88500 & 2.29630 \\
\hline $\mathrm{N}$ & -0.17230 & 6.96160 & 2.30000 \\
\hline $\mathrm{H}$ & -2.09450 & 7.62360 & 2.66900 \\
\hline $\mathrm{H}$ & -1.61520 & 3.56640 & 3.27440 \\
\hline $\mathrm{H}$ & 1.64570 & 6.05120 & 1.97590 \\
\hline $\mathrm{C}$ & 4.82560 & 0.08570 & 2.96330 \\
\hline C & 5.46840 & -1.20350 & 2.46380 \\
\hline C & 6.96230 & -1.34320 & 2.83060 \\
\hline $\mathrm{C}$ & 7.57610 & -2.16610 & 1.66170 \\
\hline $\mathrm{C}$ & 6.39650 & -2.39480 & 0.69000 \\
\hline $\mathrm{N}$ & 5.49870 & -1.28150 & 1.00380 \\
\hline $\mathrm{H}$ & 4.92250 & -2.06170 & 2.91460 \\
\hline $\mathrm{H}$ & 8.37230 & -1.59940 & 1.17250 \\
\hline $\mathrm{H}$ & 8.00720 & -3.11310 & 1.99760 \\
\hline $\mathrm{H}$ & 6.69570 & -2.36810 & -0.36260 \\
\hline $\mathrm{H}$ & 5.93980 & -3.38370 & 0.88200 \\
\hline $\mathrm{H}$ & 5.02540 & 0.21290 & 4.03870 \\
\hline $\mathrm{H}$ & 5.26250 & 0.93950 & 2.44250 \\
\hline $\mathrm{H}$ & 2.90390 & -0.78240 & 2.91330 \\
\hline $\mathrm{H}$ & 2.94550 & 4.44130 & 2.82430 \\
\hline $\mathrm{H}$ & 0.70360 & 0.15810 & 2.67250 \\
\hline $\mathrm{H}$ & 7.42500 & -0.35270 & 2.88780 \\
\hline $\mathrm{H}$ & 4.57240 & -1.38960 & 0.60090 \\
\hline $\mathrm{H}$ & 7.09210 & \multirow{2}{*}{\multicolumn{2}{|c|}{$\begin{array}{cc}-1.82320 & 3.80440 \\
\ldots . & -833.53755487587\end{array}$}} \\
\hline & & & \\
\hline
\end{tabular}

136:

angstroms

\section{$\mathbf{x}$}

C $\quad-0.29470$

C $\quad-0.95960$

\section{Y}

2.79650

3.99430
$-2.87670$

$-2.54410$ 


$\begin{array}{lrrr}\mathrm{C} & -2.20440 & 3.89300 & -1.90080 \\ \mathrm{C} & -2.77850 & 2.64840 & -1.67270 \\ \mathrm{~N} & -0.86520 & 1.56780 & -2.65100 \\ \mathrm{H} & 0.67750 & 2.85490 & -3.35080 \\ \mathrm{H} & -2.72250 & 4.76890 & -1.53350 \\ \mathrm{H} & -3.74230 & 2.58570 & -1.18140 \\ \mathrm{C} & 1.05370 & 7.64350 & -2.61680 \\ \mathrm{~N} & -0.28860 & 7.63800 & -2.80200 \\ \mathrm{C} & -1.01170 & 6.48830 & -2.92980 \\ \mathrm{C} & -0.32450 & 5.24300 & -2.75620 \\ \mathrm{~N} & 1.03470 & 5.27590 & -2.64330 \\ \mathrm{C} & 1.74220 & 6.43230 & -2.58950 \\ \mathrm{H} & 1.57910 & 8.58670 & -2.53730 \\ \mathrm{~N} & 3.08790 & 6.39720 & -2.47110 \\ \mathrm{C} & -4.99180 & 6.95750 & -4.31060 \\ \mathrm{C} & -4.26160 & 5.82770 & -4.67900 \\ \mathrm{C} & -2.94930 & 5.66060 & -4.22760 \\ \mathrm{C} & -2.35230 & 6.61410 & -3.37100 \\ \mathrm{C} & -3.09380 & 7.77160 & -3.04710 \\ \mathrm{C} & -4.40590 & 7.93190 & -3.50240 \\ \mathrm{H} & -6.00710 & 7.08490 & -4.66340 \\ \mathrm{H} & -4.70730 & 5.08620 & -5.32970 \\ \mathrm{H} & -2.67100 & 8.54350 & -2.41540 \\ \mathrm{H} & -4.96860 & 8.81510 & -3.22840 \\ \mathrm{H} & -2.39460 & 4.80440 & -4.58750 \\ \mathrm{C} & -0.13360 & 0.33960 & -3.05160 \\ \mathrm{C} & 0.09200 & 0.30090 & -4.56910 \\ \mathrm{C} & 1.19090 & 0.21110 & -2.28720 \\ \mathrm{H} & -0.72210 & -0.57070 & -2.80390 \\ \mathrm{H} & 1.66470 & -0.76880 & -2.51000 \\ \mathrm{H} & 1.90190 & 1.01540 & -2.56890 \\ \mathrm{H} & 1.00070 & 0.26670 & -1.19440 \\ \mathrm{H} & 0.52160 & -0.68030 & -4.86430 \\ \mathrm{H} & -0.87710 & 0.43090 & -5.09590 \\ \mathrm{H} & 0.78870 & 1.10040 & -4.89690 \\ \mathrm{H} & 3.55770 & 5.47980 & -2.44750 \\ \mathrm{H} & 3.61870 & 7.27990 & -2.42490 \\ \mathrm{C} & -2.10440 & 1.48960 & -2.05890 \\ \mathrm{O} & -2.63410 & 0.40600 & -1.84630 \\ & & & \end{array}$

Total energy............ $\quad-991.03775672139$ hartrees Number of imaginary frequencies: 0

(1) Bochevarov, A. D.; Harder, E.; Hughes, T. F.; Greenwood, J. R.; Braden, D. A.; Philipp, D. M.; Rinaldo, D.; Halls, M. D.; Zhang, J.; Friesner, R. A. Int. J. Quantum Chem. 2013, 113 (18), 2110-2142.

(2) Adams, N. D.; Adams, J. L.; Burgess, J. L.; Chaudhari, A. M.; Copeland, R. a.; Donatelli, C. a.; Drewry, D. H.; Fisher, K. E.; Hamajima, T.; Hardwicke, M. A.; Huffman, W. F.; Koretke-Brown, K. K.; Lai, Z. V.; McDonald, O. B.; Nakamura, H.; Newlander, K. a.; Oleykowski, C. a.; Parrish, C. a.; Patrick, D. R.; Plant, 
R.; Sarpong, M. a.; Sasaki, K.; Schmidt, S. J.; Silva, D. J.; Sutton, D.; Tang, J.; Thompson, C. S.; Tummino, P. J.; Wang, J. C.; Xiang, H.; Yang, J.; Dhanak, D. J. Med. Chem. 2010, 53 (10), 3973-4001.

Sudau, A.; Neumann, J.; Benting, J. Thienylpyri(mi)dinylpyrazole. WO 2012/175513A1, 2012.

(4) Benarous, R.; Chevreuil, F.; Ledoussal, B.; Chasset, S.; Le Strat, F. Inhibitors of viral replication, their process of preparation and their therapeutical uses. WO 2014/053665 A1, 2014.

(5) Picard, J. A.; Roth, B. D.; Sliskovic, D. R. Trans-6-(2-(N-heteroaryl-3,5-disubstituted)pyrazol-4-yl)-ethyl)or ethenyl)tetrahydro-4-hydroxypyran-2-one inhibitors of cholesterol biosynthesis. US 4957971 A, 1990.

(6) Kimura, T.; Ohkawa, N.; Nakao, A.; Nagasaki, T.; Shimozato, T. Cyclic tertiary amine compound. US 20070049620 A1, 2007.

(7) Chianelli, D.; Molteni, V.; Albaugh, P. A.; Choi, H.-S.; Loren, J.; Wang, Z.; Mishra, P. Compounds and compositions as microsomal prostaglandine Synthase-1 inhibitors. WO 2010/127152 A2, 2010.

El Borai, M.; Moustafa, A. H.; Anwar, M.; Abdel Hay, F. I. Pol. J. Chem. 1981, 55 (7-8), 1659-1665.

(9) Schwiebert, E.; Streiff, J.; Dixon, J.; Gao, H. Coumarin derivatives and methods of use in treating cystic fibrosis, chronic obstructive pulmonary disease, and misfolded protein disorders. WO 2014/152213 A2, 2014.

(10) Tamayo, N. A.; Norman, M. H.; Bartberger, M. D.; Hong, F.-T.; Bo, Y.; Liu, L.; Nishimura, N.; Yang, K. C.; Tadesse, S.; Fotsch, C.; Chen, J.; Chmait, S.; Cupples, R.; Hale, C.; Jordan, S. R.; Lloyd, D. J.; Sivits, G.; Van, G.; St. Jean, D. J. J. Med. Chem. 2015, 58 (11), 4462-4482.

(11) Forlani, L.; Medici, A.; Ricci, M.; Todesco, P. E. Synthesis 1977, 1977 (05), 320-322.

(12) Chen, Z.; Hamdouchi, C. H.; Hembre, E. J.; Hipskind, P. A.; Myers, J. K.; Toth, J. L.; Takakuwa, T. Thiazole pyrazolopyrimidines as CRF1 receptor antagonists. WO 2008/036579 A1, 2008.

(13) Hilpert, K.; Hubler, F.; Renneberg, D.; Stamm, S. Heterocyclic amide derivatives as P2X7 receptor antagonists. WO 2014/115078 A1, 2014.

(14) Zheng, M.-H.; Sun, W.; Jin, J.-Y.; Yan, C.-H. J. Fluoresc. 2014, 24 (4), 1169-1176.

(15) Takami, S.; Irie, M. Tetrahedron 2004, 60 (29), 6155-6161.

(16) Hung, D.; Serrano-Wu, M.; Grant, S.; Kawate, T. Substituted aminothiazoles for the treatment of tuberculosis. WO 2014/159938 A1, 2014.

(17) Martelletti, A.; Dietrich, H.; Hoffmann, M. G.; Helmke, H.; Dittgen, J.; Hills, M.; Kehne, H.; Rosinger, C.; Schmutzler, D.; Drewes, M. W.; Feucht, D. New thiazole derivatives useful as herbicides and plant growth 
regulators. DE 10 2007/012168 A1, 2008.

(18) Leboho, T. C.; van Vuuren, S. F.; Michael, J. P.; de Koning, C. B. Org. Biomol. Chem. 2014, 12 (2), $307-$ 315.

(19) Burger, M. T.; Pecchi, S.; Wagman, A.; Ni, Z.-J.; Knapp, M.; Hendrickson, T.; Atallah, G.; Pfister, K.; Zhang, Y.; Bartulis, S.; Frazier, K.; Ng, S.; Smith, A.; Verhagen, J.; Haznedar, J.; Huh, K.; Iwanowicz, E.; Xin, X.; Menezes, D.; Merritt, H.; Lee, I.; Wiesmann, M.; Kaufman, S.; Crawford, K.; Chin, M.; Bussiere, D.; Shoemaker, K.; Zaror, I.; Maira, S.-M.; Voliva, C. F. ACS Med. Chem. Lett. 2011, 2 (10), 774-779.

(20) Carboni, S.; Da Settimo, A.; Ferrarini, P. L.; Primofiore, G.; Livi, O.; Menichetti, V.; Del Tacca, M.; Martinotti, E.; Bernardini, C.; Bertelli, A. Eur. J. Med. Chem. 1984, 17 (2), 159-164.

(21) Song, Y.; Bridges, A. J. Macrocyclic compounds for the treatment of proliferative diseases. WO 2015/050989 A2, 2015.

(22) Esteve, C.; González, J.; Gual, S.; Vidal, L.; Alzina, S.; Sentellas, S.; Jover, I.; Horrillo, R.; Alba, J. De; Tarrasón, G.; Vidal, B. Bioorg. Med. Chem. Lett. 2015, 25 (6), 1217-1222.

(23) El Bouakher, A.; Allouchi, H.; Abrunhosa-Thomas, I.; Troin, Y.; Guillaumet, G. European J. Org. Chem. 2015, No. 16, 3450-3461.

(24) Chau, R. W.; Cullis, C. A.; Duffey, M. O.; Gipson, K. E.; Hu, Y.; Li, G.; Sintchak, M. D.; Vos, T. J. Heteroaryls and uses thereof. WO 2013/096642 A1, 2013.

(25) Han, F.; Lin, S.; Liu, P.; Liu, X.; Tao, J.; Deng, X.; Yi, C.; Xu, H. ACS Med. Chem. Lett. 2015, 6, 434-438.

(26) Shiao, M. J.; Shieh, P.; Lai, J. S. J. Chinese Chem. Soc. (Taipei, Taiwan) 1988, 35 (3), 233-236.

(27) Shiao, M. J.; Shyu, L. M.; Chen, C. F. Heterocycles 1990, 31 (3), 523-527.

(28) Kumar, V.; Dority, J. A.; Bacon, E. R.; Singh, B.; Lesher, G. Y. J. Org. Chem. 1992, 57 (25), 6995-6998.

(29) Jessen, H. J.; Schumacher, A.; Shaw, T.; Pfaltz, A.; Gademann, K. Angew. Chemie - Int. Ed. 2011, 50 (18), $4222-4226$.

(30) Meana, Á.; Rodríguez, J. F.; Sanz-Tejedor, M. A.; García-Ruano, J. L. Synlett 2003, No. 11, 1678-1682.

(31) Comins, D. L.; Lyle, R. E. J. Org. Chem. 1976, 41 (11), 2065-2066.

(32) Singh, B.; Bacon, E. R.; Lesher, G. Y.; Robinson, S.; Pennock, P.; Bode, D. C.; Pagan, E. D.; Bentley, R. G.; Connel, M. J.; Hamel, L. T.; Silver, P. J. J. Med. Chem. 1995, 38 (14), 2546-2550. 
(33) Donohoe, T. J.; Bower, J. F.; Basutto, J. a.; Fishlock, L. P.; Procopiou, P. a.; Callens, C. K. a. Tetrahedron 2009, 65 (44), 8969-8980.

(34) Oi, N.; Yamamoto, N.; Suzuki, M.; Nakatani, Y.; Suhara, T.; Cho, M.; Fukumura, T.; Higuchi, M.; Minamimoto, T.; Maeda, J.; Tokunaga, M.; Nagai, Y. [11C] and [18F] labeled 1,3-diphenyl-5-(pyrimidin-2yl)-pyridin-2(1 H)-one derivatives and their use for PET imaging of AMPA receptors. WO 2014/136210 A1, 2014.

Jones, C.; Matusiak, Z. S. Chemical compounds. WO 2007/148070 A1, 2007.

(36) Eastwood, P. R.; Gonzalez Rodriguez, J.; Bach Tana, J.; Pages Santacana, L. M.; Taltavull Moll, J.; Caturla Javaloyes, J. F.; Matassa, V. G. Imidazopyridine derivatives as JAK inhibitors. WO 2011/076419 A1, 2011.

(39) Coombs, T. C.; Tanega, C.; Shen, M.; Wang, J. L.; Auld, D. S.; Gerritz, S. W.; Schoenen, F. J.; Thomas, C. J.; Aubé, J. Bioorg. Med. Chem. Lett. 2013, 23 (12), 3654-3661.

(40) Kenyon, C. P.; Oldfield, L. C.; Van Der Westhuyzen, C. W.; Rousseau, A. L.; Parkinson, C. J. Modulation of phosphoryl transferase activity of glutamine synthetase. WO 2007/105023 A1, 2007.

(41) Wang, T.; Ng, J. S.; Webber, S. E. Novel process for the preparation of 5-amino-3H-thiazolo[4,5d]pyrimidin-2-one. US 2007/0117979, 2007.

(42) Paudler, W. W.; Jovanovic, M. V. J. Org. Chem. 1983, 48 (7), 1064-1069.

(43) Vanotti, E.; Caldarelli, M.; Disingrini, T.; Nesi, M.; Tato, M.; Vianello, P.; Menichincheri, M. Tetrahedron 2009, 65 (50), 10418-10423.

(44) Kress, T. J.; Costantino, S. M. J. Heterocycl. Chem. 1973, 10 (3), 409-410.

(45) Rossignol, E.; Youssef, A.; Moreau, P.; Prudhomme, M.; Anizon, F. Tetrahedron 2007, 63 (41), 1016910176 .

(46) Guillemont, J. E. G.; Paugam, M.; Delest, B. F. M. HIV inhibiting 5-amido substituted pyrimidines. WO 2007/113254 A1, 2007.

(47) Vlád, G.; Horváth, I. J. Org. Chem. 2002, 67 (18), 6550-6552.

(48) Du Bois, D. J.; Mao, L.; Rogers, D. H.; Williams, J. P. 2,5-substituted pyrimidine derivatives as CCR-3 receptor antagonists IX. WO 2004/004731 A1, 2004. 
(49)

(50)

Ondi, L.; Lefebvre, O.; Schlosser, M. European J. Org. Chem. 2004, No. 17, 3714-3718.

Maddess, M.; Carter, R. Synthesis 2012, 44 (07), 1109-1118.

Raynham, T. M.; Hammonds, T. R.; Gilliatt, J. H.; Charles, M. D.; Pave, G. A.; Foxton, C. H.; Carr, J. L.; Mistry, N. S. Amino-ethyl-amino-aryl (aeaa) compounds and their use. WO 2007/125331 A2, 2007.

Wang, Z.; Chi, Y.; Harris, A.; Gray, D.; Davoren, J. Synthesis 2011, 2011 (10), 1529-1531.

Suzuki, N.; Shiota, T.; Watanabe, F.; Haga, N.; Murashi, T.; Ohara, T.; Matsuo, K.; Oomori, N.; Yari, H.; Dohi, K.; Inoue, M.; Iguchi, M.; Sentou, J.; Wada, T. Bioorg. Med. Chem. Lett. 2011, 21 (6), 1601-1606.

Reigan, P.; Gbaj, A.; Stratford, I. J.; Bryce, R. a; Freeman, S. Eur. J. Med. Chem. 2008, 43 (6), 1248-1260.

Blake, J. F.; Boyd, S. A.; Cohen, F.; De Meese, J.; Fong, K. C.; Gaudino, J. J.; Kaplan, T.; Marlow, A. L.; Seo, J.; Thomas, A. A.; Tian, H.; Young, W. B. Heterobicyclic pyrazole compounds and methods of use. WO 2007/103308 A2, 2007.

Zahn, S. K.; Boehmelt, G.; Mantoulidis, A.; Reiser, U.; Treu, M.; Guertler, U.; Schoop, A.; Solca, F.; Tontsch-Grunt, U.; Brueckner, R.; Reither, C.; Herfurth, L.; Kraemer, O.; Stadtmueller, H.; Engelhardt, H. 2,4-Diamino-pyrimidine used as Aurora inhibitors. WO 2007/003596 A1, 2007.

Yonishi, S.; Aoki, S.; Matsushima, Y.; Akahane, A. Pyrazine derivatives and pharmaceutical use thereof. WO 2005/040151 A1, 2005.

Li, G.; Li, Q.; Li, T.; Lin, N.-H.; Mantei, R. A.; Sham, H. L.; Wang, G. T. Urea kinase inhibitors. US 2004/0034038 A1, 2004.

Connolly, P. J.; Lin, S.-C.; Macielag, M. J.; Zhang, Y.-M. Pyrazines as delta opioid receptor modulators. WO 2011/053696 A1, 2011.

Blaney, J.; McDonald, I.; Tomimoto, M. Compound libraries and methods for drug discovery. WO 2004/092727 A2, 2004.

Bouloc, N.; Large, J. M.; Kosmopoulou, M.; Sun, C.; Faisal, A.; Matteucci, M.; Reynisson, J.; Brown, N.; Atrash, B.; Blagg, J.; McDonald, E.; Linardopoulos, S.; Bayliss, R.; Bavetsias, V. Bioorg. Med. Chem. Lett. 2010, 20 (20), 5988-5993.

Koppitz, M.; Klar, U.; Jautelat, R.; Kosemund, D.; Bohlmann, R.; Bader, B.; Lienau, P.; Siemeister, G. Substituted 6-imidazopyrazines for use as mps-1 and tkk inhibitors in the treatment of hyperproliferative disorders. WO 2012/080234 A1, 2012.

Macleod, A.; Mitchell, D. R.; Palmer, N. J.; Parsy, C. C.; Goldsmith, M. D.; Harris, C. J. Imidazopyrazine compounds. WO 2009/024585 A2, 2009. 
(64) Senga, K.; O’Brien, D. E.; Scholten, M. B.; Novinson, T.; Miller, J. P.; Robins, R. K. J. Med. Chem. 1982, 25 (3), 243-249.

Yin, L.; Liebscher, J. Synthesis 2004, 2004 (14), 2329-2334.

(66) Brown, R. E.; Burkamp, F.; Doughty, V. A.; Fletcher, S. R.; Hollingworth, G. J.; Jones, B. A.; Sparey, T. J. Substituted amino heterocycles as vr-1 antagonists for treating pain. WO 2004/074290A1, 2004.

(67) Vogel, A.; Troxler, F. Helv. Chim. Acta 1975, 58 (3), 761-771.

(68) Wishart, N.; Argiriadi, M. A.; Calderwood, D. J.; Ericsson, A. M.; Fiamengo, B. R.; Frank, K. E.; Friedman, M.; George, D. M.; Goedken, E. R.; Josephsohn, N. S.; Li, B. C.; Morytko, M. J.; Stewart, K. D.; Voss, J. W.; Wallace, G. A.; Wang, L.; Woller, K. R. Novel tricyclic compounds. US 2011/0311474 A1, 2011.

(69) Chobanian, H. R.; Guo, Y.; Liu, P.; Chioda, M. D.; Fung, S.; Lanza, T. J.; Chang, L.; Bakshi, R. K.; Dellureficio, J. P.; Hong, Q.; McLaughlin, M.; Belyk, K. M.; Krska, S. W.; Makarewicz, A. K.; Martel, E. J.; Leone, J. F.; Frey, L.; Karanam, B.; Madeira, M.; Alvaro, R.; Shuman, J.; Salituro, G.; Terebetski, J. L.; Jochnowitz, N.; Mistry, S.; McGowan, E.; Hajdu, R.; Rosenbach, M.; Abbadie, C.; Alexander, J. P.; Shiao, L.-L.; Sullivan, K. M.; Nargund, R. P.; Wyvratt, M. J.; Lin, L. S.; DeVita, R. J. ACS Med. Chem. Lett. 2014, 5 (6), 717-721.

(70) Pattanayak, B. K.; Rout, D. N.; Mahapatra, G. N. Indian J. Chem. 1978, $16 B$ (11), 1030-1032.

(71) Zhang, M.-Z.; Chen, Q.; Mulholland, N.; Beattie, D.; Irwin, D.; Gu, Y.-C.; Yang, G.-F.; Clough, J. Eur. J. Med. Chem. 2012, 53, 283-291.

(72) Chobanian, H. R.; Lin, L. S.; Chioda, M. D.; DeVita, R. J.; Nargund, R. P.; Guo, Y.; Hamill, T.; Wenping, L.; Henze, D. A. Pharmaceutical compositions for the treatment of pain and other indications. WO 2011/094209 A1, 2011.

(73) Blaquiere, N.; Burch, J.; Castanedo, G.; Feng, J. A.; Hu, B.; Lin, X.; Staben, S.; Wu, G.; Yuen, P. Alkynyl alcohols and methods of use. WO 2015/025026 A1, 2015.

Bellina, F.; Cauteruccio, S.; Di Fiore, A.; Rossi, R. European J. Org. Chem. 2008, No. 32, 5436-5445.

Bellina, F.; Cauteruccio, S.; Di Fiore, A.; Marchetti, C.; Rossi, R. Tetrahedron 2008, 64 (26), 6060-6072.

Skogh, A.; Fransson, R.; Sköld, C.; Larhed, M.; Sandström, A. J. Org. Chem. 2013, 78 (23), 12251-12256.

Hoekstra, W. J.; Rafferty, S. W.; Yates, C. M.; Schotzinger, R. J.; Loso, M.; Sullenberger, M. Metalloenzyme inhibitor compounds. WO 2012/177603A2, 2012.

Edgar, K. J.; Falling, S. N. J. Org. Chem. 1990, 55 (18), 5287-5291. 
(79) Vidal Juan, B.; Esteve Trias, C.; Soca Pueyo, L.; Eastwood, P. R. Pyrazine derivatives useful as Adenosine Receptor Antagonists. WO 2007/017096 A1, 2007.

(80) Barlaam, B. C.; Bower, J. F.; Delouvrie, B.; Fairley, G.; Harris, C. S.; Lambert, C.; Ouvry, G.; Winter, J. J. G. Pyridine and pyrazine derivatives-083. WO 2009/053737 A2, 2009. 